Sonderdruck aus:

Mitteilungen

aus der

Arbeitsmarkt- und

Berufsforschung

Werner Karr, Karl John

Mehrfacharbeitslosigkeit und kumulative Arbeitslosigkeit 


\section{Mitteilungen aus der Arbeitsmarkt- und Berufsforschung (MittAB)}

Die MittAB verstehen sich als Forum der Arbeitsmarkt- und Berufsforschung. Es werden Arbeiten aus all den Wissenschaftsdisziplinen veröffentlicht, die sich mit den Themen Arbeit, Arbeitsmarkt, Beruf und Qualifikation befassen. Die Veröffentlichungen in dieser Zeitschrift sollen methodisch, theoretisch und insbesondere auch empirisch zum Erkenntnisgewinn sowie zur Beratung von Öffentlichkeit und Politik beitragen. Etwa einmal jährlich erscheint ein „Schwerpunktheft“, bei dem Herausgeber und Redaktion zu einem ausgewählten Themenbereich gezielt Beiträge akquirieren.

\section{Hinweise für Autorinnen und Autoren}

Das Manuskript ist in dreifacher Ausfertigung an die federführende Herausgeberin

Frau Prof. Jutta Allmendinger, Ph. D.

Institut für Arbeitsmarkt- und Berufsforschung

90478 Nürnberg, Regensburger Straße 104

zu senden.

Die Manuskripte können in deutscher oder englischer Sprache eingereicht werden, sie werden durch mindestens zwei Referees begutachtet und dürfen nicht bereits an anderer Stelle veröffentlicht oder zur Veröffentlichung vorgesehen sein.

Autorenhinweise und Angaben zur formalen Gestaltung der Manuskripte können im Internet abgerufen werden unter http://doku.iab.de/mittab/hinweise_mittab.pdf. Im IAB kann ein entsprechendes Merkblatt angefordert werden (Tel.: 09 11/1 7930 23, Fax: 09 11/1 7959 99; E-Mail: ursula.wagner@iab.de).

\section{Herausgeber}

Jutta Allmendinger, Ph. D., Direktorin des IAB, Professorin für Soziologie, München (federführende Herausgeberin)

Dr. Friedrich Buttler, Professor, International Labour Office, Regionaldirektor für Europa und Zentralasien, Genf, ehem. Direktor des IAB Dr. Wolfgang Franz, Professor für Volkswirtschaftslehre, Mannheim

Dr. Knut Gerlach, Professor für Politische Wirtschaftslehre und Arbeitsökonomie, Hannover

Florian Gerster, Vorstandsvorsitzender der Bundesanstalt für Arbeit

Dr. Christof Helberger, Professor für Volkswirtschaftslehre, TU Berlin

Dr. Reinhard Hujer, Professor für Statistik und Ökonometrie (Empirische Wirtschaftsforschung), Frankfurt/M .

Dr. Gerhard Kleinhenz, Professor für Volkswirtschaftslehre, Passau

Bernhard Jagoda, Präsident a.D. der Bundesanstalt für Arbeit

Dr. Dieter Sadowski, Professor für Betriebswirtschaftslehre, Trier

\section{Begründer und frühere Mitherausgeber}

Prof. Dr. Dieter Mertens, Prof. Dr. Dr. h.c. mult. Karl Martin Bolte, Dr. Hans Büttner, Prof. Dr. Dr. Theodor Ellinger, Heinrich Franke, Prof. Dr. Harald Gerfin,

Prof. Dr. Hans Kettner, Prof. Dr. Karl-August Schäffer, Dr. h.c. Josef Stingl

\section{Redaktion}

Ulrike Kress, Gerd Peters, Ursula Wagner, in: Institut für Arbeitsmarkt- und Berufsforschung der Bundesanstalt für Arbeit (IAB),

90478 Nürnberg, Regensburger Str. 104, Telefon (09 11) 17930 19, E-Mail: ulrike.kress@iab.de: (09 11) 1793016 ,

E-Mail: gerd.peters@iab.de: (09 11) 17930 23, E-Mail: ursula.wagner@iab.de: Telefax (09 11) 1795999.

\section{Rechte}

Nachdruck, auch auszugsweise, nur mit Genehmigung der Redaktion und unter genauer Quellenangabe gestattet. Es ist ohne ausdrückliche Genehmigung des Verlages nicht gestattet, fotografische Vervielfältigungen, Mikrofilme, Mikrofotos u.ä. von den Zeitschriftenheften, von einzelnen Beiträgen oder von Teilen daraus herzustellen.

\section{Herstellung}

Satz und Druck: Tümmels Buchdruckerei und Verlag GmbH, Gundelfinger Straße 20, 90451 Nürnberg

\section{Verlag}

W. Kohlhammer GmbH, Postanschrift: 70549 Stuttgart: Lieferanschrift: Heßbrühlstraße 69, 70565 Stuttgart: Telefon 07 11/78 63-0; Telefax 07 11/78 63-84 30: E-Mail: waltraud.metzger@kohlhammer.de, Postscheckkonto Stuttgart 16330.

Girokonto Städtische Girokasse Stuttgart 2022309.

ISSN 0340-3254

\section{Bezugsbedingungen}

Die „Mitteilungen aus der Arbeitsmarkt- und Berufsforschung“ erscheinen viermal jährlich. Bezugspreis: Jahresabonnement 52,- $€$ inklusive Versandkosten: Einzelheft 14,- $€$ zuzüglich Versandkosten. Für Studenten, Wehr- und Ersatzdienstleistende wird der Preis um $20 \%$ ermäßigt. Bestellungen durch den Buchhandel oder direkt beim Verlag. Abbestellungen sind nur bis 3 Monate vor Jahresende möglich

\section{Zitierweise:}

MittAB = „Mitteilungen aus der Arbeitsmarkt- und Berufsforschung“ (ab 1970)

Mitt $($ IAB) = „Mitteilungen“ (1968 und 1969)

In den Jahren 1968 und 1969 erschienen die „Mitteilungen aus der Arbeitsmarkt- und Berufsforschung“ unter dem Titel

„Mitteilungen“, herausgegeben vom Institut für Arbeitsmarkt- und Berufsforschung der Bundesanstalt für Arbeit.

Internet: http://www.iab.de 


\title{
Mehrfacharbeitslosigkeit und kumulative Arbeitslosigkeit
}

\author{
Werner Karr, Karl John*)
}

\begin{abstract}
Mehrfacharbeitslosigkeit, also wiederholt auftretende Arbeitslosigkeit bei der gleichen Person, und die daraus resultierende kumulative Arbeitslosigkeit können nach wie vor in periodischen Statistiken nicht erfaßt werden. Die Ermittlung ihres Umfangs, ihrer Struktur und ihrer zeitlichen Entwicklung erfolgt in eigens dafür angelegten Untersuchungen. Auch das IAB hat schon öfter Ergebnisse zur Mehrfacharbeitslosigkeit, häufig in Verbindung mit anderen Aspekten der Arbeitslosigkeit, publiziert. Die vorliegende Studie befaßt sich ausschließlich mit diesem Thema, sie basiert auf aktuellen Zahlen in vergleichsweise „langen“ Beobachtungszeiträumen. Ihre wichtigsten Ergebnisse sind:
\end{abstract}

- Im 5-Jahreszeitraum 7/1979 bis 6/1984 beträgt die durchschnittliche Mehrfacharbeitslosigkeit 1,7; dies führt bei den betroffenen Personen zu einer kumulativen Arbeitslosigkeit von 40,5 Wochen.

- Mehrfacharbeitslosigkeit kommt bei allen hier beobachteten sozio-demographischen Personengruppen vor. Die Unterschiede im Ausmaß sind nur zwischen Männern und Frauen und zwischen geringer und hoher Qualifikation beachtlich, ansonsten eher gering. Insbesondere tritt Mehrfacharbeitslosigkeit durchgängig in allen Altersgruppen auf.

- Die kumulative Arbeitslosigkeit verringert die Unterschiede zwischen verschiedenen Gruppen weiter. Sie beschreibt im Ergebnis Problemlagen am Arbeitsmarkt treffender: (1) die Qualifikation behält einen entscheidenden Einfluß, (2) die Belastung der Älteren wird erst richtig sichtbar, (3) die regionalen Arbeitsmarktlagen werden nur durch sie treffend wiedergegeben.

- Mehrfacharbeitslosigkeit und kumulative Arbeitslosigkeit haben, gemessen in gleitenden 5-Jahreszeiträumen von 1977 bis 1986, ständig zugenommen. Bei zunehmender Dauer einzelner Arbeitslosigkeitsperioden und steigender Mehrfacharbeitslosigkeit ,verdichtet“ sich diese; ihre Abfolge wird schneller bzw. die Beschäftigungszeiten zwischen Arbeitslosigkeitsperioden werden kürzer. Dies ist auch ein Indiz dafür, daß eine einmal eingetretene Arbeitslosigkeit die Wahrscheinlichkeit für weitere Arbeitslosigkeitsperioden bei derselben Person erhöht.

- Überlegungen zu methodischen Problemen zeigen, daß für die Messung von Mehrfacharbeitslosigkeit und kumulativer Arbeitslosigkeit die Länge des Beobachtungszeitraumes entscheidende Bedeutung hat. Ein zu kurz gewählter Zeitraum verzerrt ihr Niveau und ihre Struktur systematisch. Auch sollte künftig der Belastung durch kumulative Arbeitslosigkeit mehr Gewicht als der Mehrfacharbeitslosigkeit allein beigemessen werden.

\section{Gliederung}

1. Das Problem

2. Der bisherige Kenntnisstand

2.1 Datenlage

2.3 Anmerkungen zu den dargestellten Befunden

2.4 Offene Methodenfragen

3. Eigene Berechnungen zur Mehrfacharbeitslosigkeit

3.1 Mehrfacharbeitslosigkeit und kumulative Arbeitslosigkeit im Zeitraum 7/79 bis $6 / 84$

3.1.1 Zur Methode

3.1.2 Eckdaten

3.1.3 Mehrfacharbeitslosigkeit

3.1.4 Kumulative Arbeitslosigkeit

3.1.5 Mehrfacharbeitslosigkeit versus kumulative Arbeitslosigkeit

3.2 Entwicklung von Mehrfacharbeitslosigkeit und kumulativer Arbeitslosigkeit

3.2.1 Die globale Entwicklung der Mehrfacharbeitslosigkeit in Fünf-Jahres-Zeiträumen

3.2.2 Die Verteilung der Arbeitslosigkeitsperioden in der zeitlichen Entwicklung

\footnotetext{
*) Dr. Werner Karr und Karl John sind Mitarbeiter im IAB. Der Beitrag liegt in der alleinigen Verantwortung der Autoren.
}

4. Ursachen von Mehrfacharbeitslosigkeit und kumulativer Arbeitslosigkeit

4.1 Erklärungsversuche

4.2 Ursachen für Mehrfacharbeitslosigkeit in der gegenwärtigen Arbeitsmarktsituation der Bundesrepublik Deutschland

4.2.1 Saisonarbeitslosigkeit

4.2.2 Mehrfacharbeitslosigkeit bei bestimmten demographischen Gruppen

4.2.3 Destabilisierung nach Arbeitslosigkeit

5. Schlußbetrachtungen

\section{Das Problem}

Die überaus hohe und verfestigte Arbeitslosigkeit in fast allen westlichen Industriestaaten hat zu einer Vielzahl wissenschaftlicher Untersuchungen angeregt, die zwar nicht alle unmittelbar der Beseitigung dieser Arbeitslosigkeit dienten (auch nicht dazu dienen konnten), sondern viel eher auf eine gleichmäßigere Verteilung unter den Betroffenen abzielten: Insoweit nämlich, als man in diesen Untersuchungen jene beschreibbaren Personengruppen zu identifizieren suchte, die von Arbeitslosigkeit besonders stark betroffen sind, um ihnen mit geeigneten Maßnahmen aus der Arbeitslosigkeit herauszuhelfen oder diese - präventiv - erst gar nicht entstehen zu lassen.

Dem in den 70er Jahren üblicherweise verfügbaren statistischen Instrumentarium entsprechend stützte man Aussa- 
gen über Arbeitslose (gemeint waren durchaus arbeitslose Personen) auf die bekannten Bestands-Strukturen der Arbeitslosigkeit, die, aus heutiger Sicht, das Arbeitslosigkeitsvolumen einer Gruppe (der Arbeiter, der Ungelernten, der Akademiker usw.) angeben, über das Risiko für einzelne Personen (Personengruppen) zunächst aber wenig aussagen. So sind dann, neben der sehr unpräzisen Definition des Risikos „Arbeitslosigkeit“, auch häufig Fehlurteile entstanden, insbesondere, wenn die Dauer der Arbeitslosigkeit als Risikokriterium herangezogen und diese aber als bisherige Verweildauer im Arbeitslosenbestand gemessen wurde.

Im Übergang zu den 80er Jahren war man methodisch und dann auch statistisch - in der Lage, die Arbeitslosigkeit in ihrem Prozeßcharakter zu beschreiben und konnte damit sichere Aussagen über bestimmte Personengruppen (und nicht mehr nur über „Personendurchschnitte“) machen. Eine Unterscheidung in Zugangs- und Verbleibsrisiko erhellte das Feld zusätzlich.

Diese neue Darstellungsweise ließ allerdings wieder eine Vielzahl von Fehldeutungen und sogar mißbräuchlichen Interpretationen $\mathrm{zu}$ :

- Obwohl die absolute Zahl der Langfristarbeitslosen sehr hoch ist und in den letzten Jahren noch immer zugenommen hat, erschien ihr Anteil in der Prozeßbetrachtung deutlich reduziert, und das bei einer ebenfalls niedriger ausgewiesenen abgeschlossenen Dauer der Arbeitslosigkeit.

- Die große Anzahl kurzfristiger Arbeitslosigkeitsperioden, die die genannte Relativierung bewirkt, kann auch vordergründig den Anschein erwecken, als ob Arbeitslosigkeit für die Mehrzahl der Betroffenen - wegen der kurzen Dauer - ein vergleichsweise unproblematisches Ereignis wäre. Dabei wird übersehen, daß die in den Bewegungsstatistiken nachgewiesenen „Personen“ letztlich nur „Fälle“ sind, also Ereignisse, die sich im Laufe der Zeit möglicherweise ganz massiv bei bestimmten Personen konzentrieren können. Mit anderen Worten, eine Abfolge von mehreren Arbeitslosigkeitsperioden bei der gleichen Person ist vielleicht ähnlich zu beurteilen wie eine ununterbrochen lange Arbeitslosigkeitsdauer, wenn auch möglicherweise die Personengruppen, in denen das eine oder das andere sich häuft, verschieden sind und möglicherweise auch die arbeitsmarktpolitische Prophylaxe oder Therapie.

Dieses Problem ist schon länger bekannt. Methodisch wurde der Risikobegriff um das Wiederholungsrisiko erweitert und enthält damit ein Zugangs-, Verbleibs- und Wiederholungsrisiko. Allerdings verhinderte das Fehlen personenbezogener Daten in längerer zeitlicher Betrachtung eingehendere empirische Untersuchungen.

Inzwischen wird über die Mehrfacharbeitslosigkeit und die sich daraus ergebende kumulierte Arbeitslosigkeit bzw. über instabile Beschäftigungsverhältnisse, mißglückte Wiedereingliederungen usw. aller Orten diskutiert. Die Forderungen und Wünsche nach Erhebung der einschlägigen

\footnotetext{
1) Hanefeld, U., Das sozio-ökonomische Panel, Frankfurt, New York 1987

$\left.{ }^{2}\right)$ ALLBUS, Allgemeine Bevölkerungsumfrage der Sozialwissenschaften, Zentralarchiv Köln

$\left.{ }^{3}\right)$ Die Unterlagen werden 15 Monate nach Ablauf des Jahres, in dem das Bewerberangebot erledigt oder das letzte Beratungsgespräch geführt wurde, aufbewahrt.

$\left.{ }^{3 a}\right)$ Cramer, U., Wieviel Arbeitslose erhalten noch Lohnersatzleistungen?, in:
} MittAB 2/1986, S. $203 \mathrm{ff}$.
Daten nehmen national und international zu. In dieser Situation wurde auch im IAB ein weiterer Versuch unternommen, trotz großer Schwierigkeiten mit der empirischen Datenbasis, eine Abschätzung der Mehrfacharbeitslosigkeit nach einigen wichtigen Gliederungsmerkmalen vorzunehmen. Die Ergebnisse werden im folgenden vorgestellt.

\section{Der bisherige Kenntnisstand}

\subsection{Datenlage}

Die Ermittlung der Mehrfacharbeitslosigkeit und der kumulierten Arbeitslosigkeitsdauer kann sich auf individualisierte, also personengebundene Aufzeichnungen in Registern, auf Befragungen oder auf beides zusammen stützen.

Erwerbsbiographische Angaben gehören zum Standarddatensatz des seit 1983 existierenden sozioökonomischen Panels ${ }^{1}$ ), aus welchem Mehrfacharbeitslosigkeit mittlerweile auch bereits ermittelt wird. Auch im ALLBUS ${ }^{2}$ ) werden Fragen zur Arbeitslosigkeit in zurückliegenden Zeiträumen gestellt. Durch die vergleichsweise geringen Fallzahlen bei Panels, aber in der Regel auch bei anderen Stichprobenerhebungen, ist eine tiefere Merkmalsgliederung oder gar Kombination nur beschränkt sinnvoll. Geringe Fallzahlen in Verbindung mit einer breiten Fragenpalette rücken die Ergebnisse aus diesen Untersuchungsgesamtheiten schnell in die Nähe von Fallstudien. (Damit soll ihr Wert, der insbesondere in der Darstellung größerer Zusammenhänge wie Haushalt, Familie, Einkommen usw. liegt, nicht relativiert werden.)

Die Vermittlungsunterlagen der Arbeitsämter erlauben zwar einen beliebig hohen Auswahlsatz bis hin zur Totalerhebung, sie lassen auch eine ausreichende Gliederung besonderer Personengruppen unter den Arbeitslosen zu, doch haben sie wegen ihrer kurzen Aufbewahrungsfrist von 15 Monaten $^{3}$ ) für die hier untersuchte Frage nur sehr begrenzten Wert. Bei den inzwischen eingetretenen langen Perioden der Arbeitslosigkeit würde Mehrfacharbeitslosigkeit und kumulierte Arbeitslosigkeit in einem vergleichsweise kurzen Zeitabschnitt wie dem oben angegebenen systematisch untererfaßt. (Aus diesem Grunde hat die Bundesanstalt für Arbeit auch auf die weitere Erfassung der Mehrfacharbeitslosigkeit, wie in der Bestandserhebung bis September 1984 bzw. der Bewegungsanalyse Mai/Juni 1984, verzichtet.)

Eine vergleichsweise günstige Datenlage gibt es allerdings für Zahl und Dauer der mit Arbeitslosengeld und/oder Arbeitslosenhilfe finanzierten Arbeitslosigkeitsperioden pro Person. Allerdings sind diese finanzierten Perioden häufig nicht identisch mit den Arbeitslosigkeitsperioden selbst:

- So fehlen einerseits die Zeitabschnitte, in denen Arbeitslosigkeit nach Auslaufen der Leistungen fortdauert (1986 endete bei etwa $12 \%$ der Leistungsempfänger der Bezug von Arbeitslosengeld, ohne daß danach Anschlußarbeitslosenhilfe gewährt wurde). ${ }^{3 a}$ )

- Zum anderen fehlen jene Perioden ganz, bei denen von Beginn der Arbeitslosigkeit an keine Leistungsansprüche bestanden (1986 etwa 25\% der Arbeitslosigkeitsfälle).

Auch ist der Katalog persönlicher Merkmale in der Datei der Leistungsempfänger vergleichsweise klein. Die ansonsten für den beachtlichen Zeitraum ab 1976 existierende Datei der Leistungsempfänger kann also nicht ohne weiteres für die anstehende Frage herangezogen werden. 
Alle hier geschilderten Möglichkeiten der Ermittlung der Mehrfacharbeitslosigkeit und der kumulierten Arbeitslosigkeitsdauer sind in den letzten Jahren in der Bundesrepublik Deutschland genützt worden. Einige Ergebnisse daraus werden kurz vorgestellt.

\subsection{Ergebnisse}

Das IAB hat verschiedene Untersuchungen zur Mehrfacharbeitslosigkeit durchgeführt:

- Arbeitslose aus einer Bestands-Stichprobe von September 1974 wurden ein Jahr später (Herbst 1975) nach ihrem weiteren beruflichen Verbleib und nach vorausgegangenen Arbeitslosigkeitsperioden befragt. ${ }^{4}$ )

- In einer Abgänger-Stichprobe im Jahre 1976 wurde Mehrfacharbeitslosigkeit im Ein-Jahreszeitraum vor dieser Arbeitslosigkeit erhoben. ${ }^{5}$ )

- Im Herbst 1977 wurde nochmals im Rahmen einer umfangreicheren Abgänger-Stichprobe Mehrfacharbeitslosigkeit erfaßt. ${ }^{5}$ )

Die Ergebnisse zeigten eine vergleichsweise starke Konzentration von Mehrfacharbeitslosigkeit bei bestimmten Personengruppen. Insbesondere das Geschlecht (Männer hatten eine deutlich höhere Mehrfacharbeitslosigkeit als Frauen), aber auch die Qualifikation (höhere Mehrfacharbeitslosigkeit bei gering Qualifizierten), die Stellung im Beruf und das Alter (Arbeiter und Jüngere hatten eine höhere Mehrfacharbeitslosigkeit als Angestellte und Ältere) unterschieden den Umfang der Mehrfacharbeitslosigkeit deutlich.

Die zuletzt erwähnte Stichprobe war zusammen mit weiteren repräsentativen Gesamtheiten (Arbeitslosenbestand und Beschäftigtenbestand) Datenbasis für eine umfangreiche Untersuchung zur kumulativen Arbeitslosigkeit $\left.{ }^{6}\right)$. Sie wurde beobachtet bzw. gemessen in dem sich an die eingetretene Arbeitslosigkeit anschließenden Vier-Jahreszeitraum 1978 bis 1982 und durch mehrfache Befragung der Probanden ermittelt. Auch die Autoren dieser Studie kommen zu dem Ergebnis, ,daß auch die Chancen der längerfristigen berufsbiographischen Bewältigung von Arbeitslosigkeit durch die Betroffenen ungleich verteilt sind“.7) Nach dem Befund der Studie ergibt sich die Differenzierung aber dadurch, daß die eingetretene Arbeitslosigkeit

\footnotetext{
$\left.{ }^{4}\right)$ Brinkmann, C, Arbeitslosigkeit und Mobilität, in: MittAB 2/1977, insbesondere S. 207

$\left.{ }^{5}\right)$ IAB-Kurzberichte vom 29.7.1977, in: Kurzberichte 1976-1979, BeitrAB Nr. 42, S. 100-103, sowie Egle, F., Karr, W., Leupoldt, R., Strukturmerkmale der Arbeitslosen für Ströme und Bestände sowie Analyse der Vermittlungstätigkeit, in: MittAB 1/1980, S. 108/109

$\left.{ }^{6}\right)$ Büchtemann, C.F., Rosenbladt, B. von, Kumulative Arbeitslosigkeit, in: MittAB 3/1983, S. 262 ff.

7) Büchtemann, C.F., Rosenbladt, B. von, a.a.O., S. 267

${ }^{8}$ ) Karr, W., Anmerkungen zur Arbeitslosigkeit in der nunmehr 10 Jahre dauernden Beschäftigungskrise, in: MittAB 3/1983, S. 276 ff.

9) Andreß, H.-J., Recurrent Unemployment - The West-German Experience, Manuskript, August 1988; derselbe, Instabile Erwerbskarrieren und Mehrfacharbeitslosigkeit. Ein Vergleich mit der Problemgruppe der Langzeitarbeitslosen, in diesem Heft

${ }^{10}$ ) Andreß, H.-J., a.a.O., S. 8

$\left.{ }^{11}\right)$ Andreß, H.-J., a.a.O., S. 32

${ }^{12}$ ) OECD, Employment Outlook, September 1985, insbesondere: Moving in and out of unemployment: The incidence and patterns of recurrent unemployment in selected OECD-Countries, S. $99 \mathrm{ff}$.

${ }^{13}$ ) OECD, a.a.O., S. 100

${ }^{14)}$ OECD, a.a.O., S. 102
}

an sich bereits das Hauptrisiko für erneute Arbeitslosigkeit darstellt. Man findet demnach all jene Gruppen, die bereits ein höheres Zugangsrisiko in die Arbeitslosigkeit haben, auch mit Mehrfacharbeitslosigkeit behaftet (Grundgesamtheit Erwerbspersonen); betrachtet man dagegen den weiteren erwerbsbiographischen Weg von Arbeitslosen, unterscheiden personenbezogene Merkmale im Hinblick auf das Auftreten von Mehrfacharbeitslosigkeit nicht mehr nennenswert.

Bei den Arbeitslosen wurde aber nicht nur ihr weiteres berufliches Schicksal beobachtet, sie wurden auch nach der Gesamtdauer der in den letzten 10 Jahren (1973 bis 1982) erlebten Arbeitslosigkeit befragt. Diese betrug (für Abgänger) im Durchschnitt 12,7 Monate. Sie unterscheidet sich nachhaltig von der in diesem Zeitraum üblichen Dauer einer einzelnen Arbeitslosigkeitsperiode.

Die zuletzt genannten Befragungsergebnisse stimmen weitgehend überein mit einer Schätzung des IAB zur kumulierten Dauer der Arbeitslosigkeit und zur Mehrfacharbeitslosigkeit im Zeitraum 1974 bis $1983^{8}$ ). Nach ihr betrug die kumulierte Dauer etwa 50 Wochen bei einer Mehrfacharbeitslosigkeit von durchschnittlich 2,6. Eine Differenzierung nach bestimmten Personengruppen war damals allerdings nicht möglich.

Mit der bereits erwähnten Datenbasis (Stichprobe von Arbeitslosen aus dem Bestand und aus den Abgängen im März 1977) erfolgte eine weitere Untersuchung zur Mehrfacharbeitslosigkeit ${ }^{9}$ ). Auch in dieser Studie wird auf den vergleichsweise geringen Beitrag von personenbezogenen Merkmalen zur Erklärung von Mehrfacharbeitslosigkeit und auf den hohen Beitrag erwerbsbiographischer Merkmale hingewiesen: „A final conjecture supposes therefore that long unemployment spells can be much better explained by personal characteristics, while recurrent unemployment is better predicted by job characteristics as indicators for secondary labor markets“. ${ }^{10}$ ) ,Among the personal characteristics only vocational training achieves statistical significance in a multivariate model. Characteristics of the job like status, income, duration, and type of job termination outweigh this effect in number and magnitude. The best predictor of recurrent unemployment is duration of the last job held by the individual before unemployment in 1977“.11 ) (Siehe dazu auch Andreß, Kapitel 5, in diesem Heft.)

Die OECD hat in ihrem employment-outlook von 1985 der Mehrfacharbeitslosigkeit bzw. der kumulativen Arbeitslosigkeit ein eigenes Kapitel gewidmet ${ }^{12}$ ). Wie viele andere Experten erwarteten auch die Fachleute von der OECD eine Häufung der Mehrfacharbeitslosigkeit bei bestimmten Gruppen: „Different demographic groups seem likely to display a different propensity to recurrent unemployment. For example, it is often argued that multiple spells are concentrated among young people as a consequence of their high job turnover rate" ${ }^{13}$ ) Einige Überraschungen lösen dann aber die Befunde verschiedener Staaten (Australien, Schweden) aus, nach welchen jüngere Arbeitslose eher unterproportional von Mehrfacharbeitslosigkeit betroffen sind: „In 1983 teenagers and persons aged 55 or over who experienced unemployment in Australia and Sweden were less likely to suffer multiple spells than persons aged 20 to 54 years“. ${ }^{14}$ )

Die OECD erklärt diese Fakten mit einer atypischen Datenlage in 1983 und untermauert die eingangs erwähnte Hypothese mit älteren Daten aus den frühen 70er Jahren. 
Das Zentralarchiv Köln ermittelte im Rahmen des ALLBUS 1986, daß „,von den berufstätigen Befragten ...“ insgesamt $13,6 \%$ in den letzten 10 Jahren vor den Erhebungszeitpunkten wenigstens einmal arbeitslos (waren). ${ }^{15}$ ) Zur Entwicklung wird angeführt: „Während 1980 11,1\% der berufstätigen Befragten Arbeitslosigkeitsbetroffenheit angaben, stieg diese Anzahl über 12,1\% (1982) und 13,9\% (1984) auf 17,2\% im Jahre 1986“16).

Auf der Basis des sozioökonomischen Panels erhebt das DIW die Mehrfacharbeitslosigkeit bzw. die kumulative Arbeitslosigkeit für den Zeitraum 1984 bis $1987^{17}$ ). Dabei wird an den Erwerbslosenbestand (des Panels) im Jahre 1984 angeknüpft und die weitere Erwerbsbiographie beobachtet. Es zeigt sich, daß nur etwas weniger als die Hälfte der Erwerbslosen bis 1987 eine stabile berufliche Wiedereingliederung schafften. $31 \%$ waren immer noch oder wieder $(14,6 \%$ zu 16,2\%) arbeitslos: „Neben der Dauererwerbslosigkeit existiert jedoch in etwa gleichem Umfang das Problem der instabilen beruflichen Wiedereingliederung“"18). „Von rd. 2,4 Mio. Erwerbslosen im Jahre 1984 (erste Befragung) fand nur jeder zweite bis 1987 (vierte Befragung) dauerhaft einen Arbeitsplatz"199).

Der Autor der DIW-Studie hat in einer weiteren, differenzierteren Untersuchung auch das unterschiedliche Ausmaß der Mehrfacharbeitslosigkeit bei verschiedenen Untersuchungsgesamtheiten nachgewiesen: „Während die Quoten für Mehrfacharbeitslosigkeit bei Zugängen in Arbeitslosigkeit am niedrigsten sind, haben sie bei den Abgängen bis zur Viertbefragung ein um rd. 2 Prozentpunkte höheres Niveau. Die mit Abstand höchsten Risiken einer erneuten Arbeitslosigkeitsmeldung weisen die Bestände auf. Hier beträgt der Anteil der Mehrfachbetroffenheit zwischen $57 \%$ und $67 \% \%^{620}$ ). Es wird weiter festgestellt, daß die Mehrfacharbeitslosigkeit bei Männern höher sei als bei Frauen, sich dies aber über die kumulierte Dauer, die für beide bei 10 bis 12 Monaten liegt, wieder weitgehend ausgleiche $^{21}$ ).

Für den Arbeitsamtsbezirk Linz (Österreich) ermittelt Steiner im Zeitraum 1983/4 bis 1986/3 eine Mehrfacharbeitslosigkeit von $1,7^{22}$ ). Bei einer Unterscheidung nach den Merkmalen Geschlecht, Alter, Nationalität, Ausbildung und Berufsgruppen kommt er zu dem Ergebnis, daß ,die Unterschiede zwischen den einzelnen Gruppen ... auch hier relativ gering (sind) ${ }^{، 23}$ ). Resümierend hält er fest: „Entgegen gängigen Vorstellungen ist häufige Betroffenheit von Arbeitslosigkeit nicht auf die typischen ,Problemgruppen' am Arbeitsmarkt beschränkt, auch erwachsene Männer und Personen mit abgeschlossener Lehre sind stark davon betroffen“르).

\footnotetext{
${ }^{15}$ ) Terwey, M., in ZA-Information 21, S. 15, 16

derselbe, S. 17

$\left.{ }^{7}\right)$ Schupp, J., Trotz Anstiegs der Beschäftigung wurde Wiedereingliederung Erwerbsloser schwieriger. Ergebnisse einer Längsschnittstudie für die Jahre 1984-1987, DIW-Wochenbericht 32/88, S. $409 \mathrm{ff}$.

${ }^{18}$ ) ebenda, S. 413

) ebenda, S. 416

${ }^{20}$ ) Schupp, J., Dynamische Aspekte bei der Beobachtung von Arbeitslosigkeit, Manuskript, 15.9.1988, S. 16

$\left.{ }^{21}\right)$ ebenda, S. 17

$\left.{ }^{22}\right)$ Steiner, V., Kumulative Arbeitslosigkeit; in: Wirtschaft und Gesellschaft, 13. Jahrgang, Heft 1, 1988, S. $95 \mathrm{ff}$

${ }^{23}$ ) ebenda, S. 112

${ }^{24}$ ) ebenda, S. 120

${ }^{25}$ ) derselbe, Individuelle Arbeitslosigkeit und zukünftige Arbeitsmarktbiographie, Manuskript 1988, S. 19
}

In einer weiteren Studie untersucht Steiner den Zusammenhang zwischen der Dauer einer Arbeitslosigkeitsperiode und der sich möglicherweise anschließenden weiteren Arbeitslosigkeit: „Die Hypothese, daß langfristige Arbeitslosigkeit zu häufiger zukünftiger Betroffenheit von Arbeitslosigkeit führt, konnte empirisch nicht bestätigt werden“棌).

\subsection{Anmerkungen zu den dargestellten Befunden}

Die hier - im wesentlichen für die Bundesrepublik - vorgestellten exemplarischen Aussagen zur Mehrfacharbeitslosigkeit haben im Grunde nur eines gemeinsam: daß sie in ihren quantitativen Ergebnissen nicht miteinander verglichen werden können. Dies liegt daran, daß „Mehrfacharbeitslosigkeit" noch eher ein umgangssprachlicher Ausdruck als ein wissenschaftlicher Begriff ist, mit allen Freiheiten und Mehrdeutigkeiten im Entwurf von Meßkonzepten.

Die Studien sind im wesentlichen unter folgenden Gesichtspunkten verschieden:

- Länge des Beobachtungszeitraumes (1 Jahr, 2 Jahre, 3 Jahre, 31/2 Jahre, 4 Jahre, 10 Jahre),

- Lage des Beobachtungszeitraumes (1976, 1978 bis 1982, 1974 bis 1983, 1984 bis 1987 usw.),

- Grundgesamtheiten (Erwerbspersonen, Arbeitslosenzugänge, Arbeitslosenabgänge, Arbeitslosenbestände),

- Beobachtungsrichtung (retrospektiv oder prospektiv).

Unabhängig von diesen, die Quantitäten mit Sicherheit tangierenden Problemen sind in den Untersuchungen einige qualitative Aspekte und Tendenzen auszumachen, die vielleicht doch auf einen gemeinsamen Nenner gebracht werden können:

- Häufig und (zeitlich) zunehmend wird die Meinung vertreten, Mehrfacharbeitslosigkeit sei nicht so sehr in der Konstellation persönlicher Merkmale zu suchen, sondern im Ereignis Arbeitslosigkeit an sich. Ausnahmen sind, daß Männer in der Regel häufiger Arbeitslosigkeit hinnehmen müssen als Frauen; weiterhin, daß gering Qualifizierte höhere Mehrfacharbeitslosigkeit erfahren als Qualifizierte (im Gegensatz dazu steht aber die Österreich-Studie).

- Frühere Untersuchungen in vergleichbar kurzen Zeiträumen (IAB 1974/75, 1976, 1977) zeigten dagegen eine deutliche Unterscheidung nach Personengruppen. Auch die OECD untermauerte ihre Hypothese der unterschiedlichen demographischen Risikogruppen mit älterem Material, nachdem sie in den Daten von 1983 keine durchgängige Unterscheidung mehr treffen konnte.

Beide Befunde könnten darauf hindeuten, daß bei lange andauernden Arbeitsmarktkrisen die sich zunächst in Personenmerkmalen manifestierenden Risiken des Zugangs und der Verweildauer in Arbeitslosigkeit allmählich übergingen in ein erwerbsbiographisches De-Stabilisierungsrisiko, in dem demographische, bildungs- und qualifikationsbezogene Merkmale immer geringere Bedeutung haben; die Unterschiede können aber z.T. auch statistische Artefakte sein, die sich aus den vergleichsweise kurzen Untersuchungszeiträumen der siebziger Jahre gegenüber den inzwischen deutlich erweiterten Beobachtungszeiträumen ergeben. Darauf wird im folgenden Abschnitt kurz eingegangen. 


\subsection{Offene Methodenfragen}

Es ist leicht einzusehen, daß die bei ein und derselben Person wiederholt auftretende Arbeitslosigkeit um so häufiger beobachtet und gemessen werden kann, je länger der Zeitraum ist, der für die Beobachtung zur Verfügung steht. Damit ist unmittelbar klar, daß die Mehrfacharbeitslosigkeit als Verhältnis von Arbeitslosigkeits-Fällen zu arbeitslosen Personen keine konstante Größe ist, sondern mit der Länge des Beobachtungszeitraumes variiert.

Weiterhin ist leicht einzusehen, daß bei verschiedenen sozio-demographischen Gruppen unter den Arbeitslosen, die sich vor allem im Hinblick auf die durchschnittliche Dauer ihrer Arbeitslosigkeit unterscheiden (Ältere versus Jüngere; Frauen versus Männer), sich unterschiedliche Werte von Mehrfacharbeitslosigkeit allein dadurch ergeben, daß in einem gegebenen Zeitraum der Beobachtung kurze Arbeitslosigkeitsperioden häufiger auftreten können als lange.

Diese formale Restriktion gilt grundsätzlich. Sie ist unabhängig von der Länge des Beobachtungszeitraumes. Bei kurzen Zeiträumen (kurz im Sinne der Relation Beobachtungszeitraum zur durchschnittlichen Arbeitslosigkeitsdauer) muß angenommen werden, daß Ergebnisse zur Mehrfacharbeitslosigkeit für verschiedene Gruppen von Arbeitslosen systematisch verzerrt sind. Bei langen Zeiträumen mag die Praxisrelevanz dieses Einflusses gering werden.

Da der formale Zusammenhang zwischen möglicher Mehrfacharbeitslosigkeit in einem gegebenen Zeitraum in Abhängigkeit von der durchschnittlichen Dauer der Arbeitslosigkeit und evtl. auch der Streuung (noch) nicht bekannt ist, werden im folgenden einige Berechnungen vorgestellt, bei denen Lage und Länge des Beobachtungszeitraumes variiert werden.

Datenbasis ist die bereits erwähnte Datei der arbeitslosen Leistungsempfänger, mit der das Niveau der Mehrfacharbeitslosigkeit mit Sicherheit unterschätzt wird. Andererseits kann mit den in ihr enthaltenen Individualdaten der Prozeßcharakter der Arbeitslosigkeit in allen formalen Aspekten, also auch im Hinblick auf Mehrfacharbeitslosigkeit, voll dargestellt werden.

In Tabelle 2 wird die Mehrfacharbeitslosigkeit in EinJahreszeiträumen ermittelt. Es wird dabei die Vermutung geprüft, daß die in den achtziger Jahren stark angestiegene Dauer der Arbeitslosigkeit, die sich auch in einer Ausweitung der Leistungsbezugszeiten niederschlägt, die Mehrfacharbeitslosigkeit nicht ansteigen läßt bzw. sie sogar noch vermindert. Dies wird in den Ergebnissen bestätigt. Die Mehrfacharbeitslosigkeit beträgt 1977 1,19, sie steigt bis 1981 auf 1,20 und geht dann bis 1986 auf 1,17 zurück. Die Dauer des Leistungsbezuges pro Fall entwickelt sich gerade umgekehrt. Sie beträgt 1977 16,4 Wochen, geht dann leicht zurück und steigt schließlich auf 26,6 Wochen in 1986 an. Beide Größen (Mehrfacharbeitslosigkeit und Dauer der Arbeitslosigkeit) korrelieren mit $r=-0,66$ negativ miteinander.

In Tabelle 3 wird Mehrfacharbeitslosigkeit in gleitenden 5Jahreszeiträumen dargestellt. Sie beträgt für den Zeitraum 1/1977 bis 12/1981 1,68 und erhöht sich kontinuierlich auf 1,76 für den Zeitraum 1/1982 bis 12/1986. Damit wird die Vermutung bestätigt, daß in zu kurz gewählten Beobachtungszeiträumen die Entwicklung, aber auch das Niveau der Mehrfacharbeitslosigkeit, falsch ermittelt wird.
In Tabelle 4 schließlich wird Mehrfacharbeitslosigkeit für sukzessive ausgeweitete Zeiträume von einem Jahr (1/77 bis $12 / 77)$ bis zehn Jahre (1/77 bis $12 / 86)$ ausgewiesen. Hier werden also die Einflüsse aus der Verlängerung des Zeitraumes und aus der Verschiebung zusammen wirksam.

Entsprechend stark bzw. schnell verändert sich die Mehrfacharbeitslosigkeit von 1,19 im Jahreszeitraum 1977 auf 1,98 im Zehnjahreszeitraum 1977 bis 1986.

Aus diesen Beobachtungen ergibt sich unmittelbar auch, daß offensichtlich nicht die absolute Länge des Beobachtungszeitraumes, sondern vielmehr die Relation, in der die durchschnittliche Dauer einer Arbeitslosigkeitsperiode zu diesem Zeitraum steht, für das Ausmaß von Mehrfacharbeitslosigkeit von entscheidender Bedeutung ist (Tabelle 2). Da wir wissen, daß die durchschnittliche Dauer der Arbeitslosigkeit für unterschiedliche sozio-demographische Gruppen verschieden lang ist und sich durchaus um den Faktor 2 unterscheiden kann, ist auch - wie schon erwähnt - zu erwarten, daß für solche Gruppen eine unterschiedliche Mehrfacharbeitslosigkeit ermittelt wird, allein aus einem nicht hinreichend groß gewählten Zeitraum. Viele der früheren Befunde aus Zeiträumen von einem oder zwei Jahren dürften deshalb systematisch verzerrt sein; und zwar verzerrt in der Richtung, daß Gruppen mit durchschnittlich kurzer Verweildauer eine höhere Mehrfacharbeitslosigkeit aufweisen als solche mit langer Verweildauer.

Ein völlig anderes, bisher aber auch kaum aufgegriffenes methodisches Problem ist das der Erhebungsrichtung. Die vorstehend geforderte Verwendung langer Zeiträume für die Beobachtung und Messung von Mehrfacharbeitslosigkeit ist für aktuelle Untersuchungen oft ein Hinderungsgrund, den langdauernden Reintegrationsprozeß einer $\mathrm{Zu}$ oder Abgangskohorte von Arbeitslosen zu verfolgen. Häufig wird deshalb die retrospektive Befragung angewendet. Die mit der Retrospektivbefragung zusammenhängenden Probleme sollen hier aber nicht weiter erörtert werden. Vielmehr erscheint es wichtig, einige andere methodische Konsequenzen aus empirischen Befunden zu ziehen bzw. auf sie aufmerksam zu machen: Ein Grund für wiederholt auftretende Arbeitslosigkeit wird häufig in der bereits eingetretenen Arbeitslosigkeit gesehen, die für viele der Betroffenen die De-Stabilisierung der Erwerbsbiographie einleitet. Wenn diese Hypothese richtig ist, ist es nicht mehr unerheblich, ob Mehrfacharbeitslosigkeit prospektiv oder retrospektiv gemessen wird. Insoweit nämlich, als eine Arbeitslosen-Kohorte in prospektiver Beobachtung wegen der bereits eingetretenen Arbeitslosigkeit - höhere Mehrfacharbeitslosigkeit zu erwarten hätte als bei retrospektiver (die gegenwärtige Periode wird ja für viele von ihnen die erste sein).

Leider ist die hier häufig verwendete Datei der Leistungsempfänger zur Erhellung dieses Sachverhaltes nicht sehr geeignet. Denn die leistungsrechtlichen Restriktionen (Anspruchserschöpfung, kein neuer Anspruch bei erneuter Arbeitslosigkeit) wirken sich einseitig auf der prospektiven Seite der Beobachtungszeiträume aus. Eine Prüfung der Hypothese mit diesen Individualdaten ist daher wenig sinnvoll.

Die in einem späteren Abschnitt beschriebene Entwicklung der Mehrfacharbeitslosigkeit in gleitenden Zeiträumen deutet aber - ohne daß die Vermutung im strengen Sinne bewiesen wäre - darauf hin, daß Mehrfacharbeitslosigkeit nicht nur in der Zeit, sondern auch bei den gleichen 
Personen zunimmt, daß also im Zusammenhang mit Arbeitslosigkeit nicht nur im Hinblick auf Zugangsrisiken und Verweilrisiken, sondern auch „entlang der Zeit“" selektiert wird.

Bei künftigen Untersuchungen zur Mehrfacharbeitslosigkeit sollte dies stärker beachtet werden.

\section{Eigene Berechnungen}

\subsection{Mehrfacharbeitslosigkeit und kumulative Arbeitslosigkeit im} Zeitraum $7 / 79$ bis $6 / 84$

\subsubsection{Zur Methode}

Eine umfangreiche Erhebung bei repräsentativ ausgewählten arbeitslosen Personen über den Umfang der Mehrfacharbeitslosigkeit und das Ausmaß kumulativer Arbeitslosigkeit war aus Kapazitätsgründen sowohl bei den Arbeitsämtern als auch im IAB nicht durchzuführen. Eine Auswertung der Vermittlungsunterlagen kam wegen der zu kurzen Aufbewahrungsfristen ebenfalls nicht in Betracht.

Damit blieb letztlich nur die Möglichkeit, die Mehrfacharbeitslosigkeit mit Hilfe der in der Bundesanstalt für Arbeit verfügbaren Dateien, also insbesondere mit den Dateien über Leistungsempfänger und Beschäftigte, statistisch zu ermitteln. Es war von vornherein klar, daß in weiten Bereichen mit Schätzungen gearbeitet werden mußte und daß deshalb die Ergebnisse mit Unscharfen behaftet sind. Dem steht andererseits gegenüber, daß die Masse des Datenmaterials - immerhin werden mehrere Millionen Fälle in die Berechnungen einbezogen - eine andere Qualität der Ergebnisse verbürgt, als viele der zitierten Untersuchungen, in denen teilweise nur etwa 1000 Fälle nach mehreren Merkmalen fast zu Fallstudien ,zergliedert“ wurden.

Für folgende Fragen, die ähnlich auch anderen Orts (z.B. von der OECD) gestellt werden, sollen Antworten gefunden werden:

- Auf wieviele Fälle und auf wieviele Personen verteilt sich das Arbeitslosigkeitsvolumen einer gegebenen Periode (Mehrfacharbeitslosigkeit)?

- Wie unterscheidet sich die Dauer der Arbeitslosigkeit pro Fall von der pro Person (kumulative Arbeitslosigkeit)?

- Gibt es beschreibbare Personengruppen, bei denen Mehrfacharbeitslosigkeit besonders häufig und / oder kumulative Arbeitslosigkeit besonders umfangreich ist?

- Gibt es einen Zusammenhang derart, daß eine einmal eingetretene Arbeitslosigkeit mit hoher Wahrscheinlichkeit eine weitere Arbeitslosigkeit nach sich zieht?

Die Vorgehensweise sei hier grob skizziert:

- Ausgangspunkt ist das Volumen der Arbeitslosigkeit, gemessen in Personenjahren, im Fünf-Jahreszeitraum 7/1979 bis 6/1984.

- Innerhalb dieses Volumens wird das durch Arbeitslosengeld und Arbeitslosenhilfe finanzierte Volumen nach Personen, Fällen und Dauer ermittelt.

- Für diese Personen werden nichtfinanzierte Perioden und Teilperioden aus der Beschäftigtenstatistik dazugeschätzt. Damit ergibt sich das Arbeitslosigkeitsvolumen und seine Komponenten (Personen, Fälle, Dauer) für alle die Personen, die im Beobachtungszeitraum mindestens einmal Leistungen bezogen haben.
- Der übrige Teil des Arbeitslosigkeitsvolumens wird im gleichen Verhältnis auf Personen, Fälle und Dauer aufgeteilt. (Es sind jene Arbeitslosen, die im Beobachtungszeitraum nie irgendeine Leistung erhalten haben.) Dieser Rechengang wird separat durchgeführt für alle Gruppen, für die identische Merkmale in den verschiedenen Statistiken verfügbar sind:

- Geschlecht

- Berufsbereiche

- Qualifikation

- Altersgruppen

- LAA-Bezirke.

(Es sei ausdrücklich nochmals betont, daß die Aufteilung des Arbeitslosigkeitsvolumens der Nichtleistungsempfänger, über die ja keine weiteren Informationen vorliegen, gruppenspezifisch erfolgt. Das heißt z.B., daß das Volumen jüngerer Nicht-Leistungsempfänger auf Personen, Fälle und Dauer aufgeteilt wird wie das der jüngeren Leistungsempfänger usw.).

\subsubsection{Eckdaten}

Aus den Monatsendbeständen der Monate 7/1979 bis 6/1984 wurde ein Arbeitslosigkeitsvolumen von insgesamt 7.819.000 Personenjahren errechnet. Die Zugänge (= Fälle) belaufen sich in diesem Zeitraum auf 17.159.000. Damit ergibt sich für den Beobachtungszeitraum als durchschnittliche Dauer eines Arbeitslosigkeitsfalles eine Zeitspanne von 23,7 Wochen.

Aus der Datei der Leistungsempfänger wurden 7.875.000 verschiedene Personen ermittelt, die während ihrer individuellen Arbeitslosigkeit mindestens eine Periode bzw. eine Teilperiode Arbeitslosengeld und/oder Arbeitslosenhilfe bezogen haben. Das finanzierte Arbeitslosigkeitsvolumen dieser Personen betrug 5.255.000 Personenjahre, das nichtfinanzierte Volumen der gleichen Personen wurde auf 870.000 Personenjahre geschätzt. Das Arbeitslosigkeitsvolumen der Leistungsempfänger betrug damit 6.125.000 Personenjahre.

Damit verbleibt für die Arbeitslosen, die im genannten Zeitraum nie eine Leistung bezogen haben, ein Volumen von 1.694.000 Personenjahren. Legt man (mangels jeder anderen Information) die gleiche Relation zwischen Volumen und Personen bei den Arbeitslosen ohne Leistungsbezug zugrunde wie bei den Leistungsbeziehern, ergibt sich eine Personenzahl von 2.178.000.

Damit verteilt sich das eingangs erwähnte Volumen von 7.819.000 Personenjahren auf etwa 10.053.000 Personen. Die Mehrfacharbeitslosigkeit beträgt also für den genannten Fünf-Jahreszeitraum 1,71 und die kumulierte Dauer der Arbeitslosigkeit durchschnittlich 40,4 Wochen pro Person.

Diese Berechnungen wurden für alle hier aufgeführten Teilgesamtheiten separat durchgeführt (siehe folgende Seite).

\subsubsection{Die Mehrfacharbeitslosigkeit}

In den eingangs zitierten Untersuchungen wurde festgehalten, daß personenbezogene Merkmale tendenziell nicht so ausgeprägt wie erwartet das Ausmaß der Mehrfacharbeitslosigkeit beeinflussen. Ausnahmen bildeten das Geschlecht, die Qualifikation und eher nachrangig das Alter. In Berufen mit hoher Saisonarbeitslosigkeit wurde ebenfalls höhere Mehrfacharbeitslosigkeit erwartet. 
(in Tsd.)

1. Volumen der Arbeitslosigkeit im Zeitraum $7 / 79$ bis $6 / 84$

2. finanziertes Volumen der LE 5255

3. nichtfinanziertes Volumen der LE 870

4. Arbeitslosigkeitsvolumen der Leistungsempfänger $(2+3)$

5. Arbeitslosigkeitsvolumen der Nicht-Leistungsempfänger (1 minus 4)

6. Leistungsempfänger (Personen)

7. Nicht-Leistungsempfänger (Personen) $=\frac{\text { Vol. Nicht-LE (5) }}{\text { Alo. Vol. LE (4) }} \cdot$ LE Personen (6)

8. Arbeitslose Personen $(6+7)$

9. Arbeitslosen-Zugänge (Fälle)

10. Mehrfacharbeitslosigkeit $(9: 8)$

11. Dauer pro Person (1:8) $\cdot 52$ in Wochen

12. Dauer pro Fall (1:9) $\cdot 52$ in Wochen

Leider enthält die Datei der Leistungsempfänger nur wenige personenbezogene Merkmale bzw. teilweise solche, die sich in anderen Statistiken, die zur Ergänzung benötigt werden, nicht mehr finden (beispielsweise den Familienstand).

Als für diese Untersuchung relevante Merkmale kommen in Betracht:

- das Geschlecht

- das Alter

- der Beruf

- die Region.

Das Merkmal Beruf ist für sich allein betrachtet vielleicht nicht einmal so sehr interessant; verschiedene, nach Berufen geordnete Teilgesamtheiten erlauben jedoch Schlüsse auf andere Sachverhalte: So findet man in den Berufsbereichen der Land- und Forstwirtschaft überwiegend Saisonarbeitslose, bei denen eine hohe Mehrfacharbeitslosigkeit zu erwarten ist, die ja hier fast zur Erwerbsbiographie gehört und mit Sicherheit anders $\mathrm{zu}$ beurteilen ist als sonstige instabile Beschäftigungsverhältnisse. Die Fertigungsberufe einerseits und die Dienstleistungsberufe andererseits geben grobe Hinweise auf die Stellung im Beruf. Fertigungsberufe werden nämlich überwiegend von Arbeitern, Dienstleistungsberufe überwiegend von Angestellten ausgeübt. Freilich sagt dies noch nichts über die Qualifikation aus, da in beiden Bereichen gering- und hochqualifizierte Beschäftigte bzw. Arbeitslose zu finden sind. Um auch ein halbwegs sicheres Urteil über Mehrfacharbeitslosigkeit in Abhängigkeit von der Qualifikation zu gewinnen, wurden jene Fertigungsberufe und jene Dienstleistungsberufe mit einem Qualifizierten-Anteil von mindestens $85 \%$ und jene mit einem Qualifizierten-Anteil von unter 30\% zusammengefaßt.

\footnotetext{
${ }^{26}$ ) Brinkmann, C., Arbeitslosigkeit und Mobilität, in: MittAB 2/1977, insb. S 207; Brinkmann, C., Verbleib und Vermittlungsprobleme von Arbeitslosen, in: MatAB 5/1983, S. 6
}

Dies führt zu folgenden Gruppen:

1. Fertigungsberufe (geringe Qualifikation)

2. Dienstleistungsberufe (geringe Qualifikation)

3. Fertigungsberufe (hohe Qualifikation)

4. Dienstleistungsberufe (hohe Qualifikation)

Die Ergebnisse zur Mehrfacharbeitslosigkeit sowie zur durchschnittlichen Dauer pro Fall und pro Person sind in Tabelle 1 zusammengestellt.

- Die Männer haben mit durchschnittlich 1,86 eine deutlich höhere Mehrfachbetroffenheit als die Frauen mit 1,52.

- Die land- und forstwirtschaftlichen Berufe sowie Bergleute und Mineralgewinner haben eine überdurchschnittliche Mehrfacharbeitslosigkeit. Viele Beschäftigungsverhältnisse werden dort durch Perioden saisonaler Arbeitslosigkeit unterbrochen. Entsprechend hohe Werte findet man in den Landesarbeitsamtsbezirken mit hohen Beschäftigtenanteilen im Fremdenverkehr (Schleswig-Holstein/Hamburg, Bayern, Baden-Württemberg) und/oder witterungsabhängigen Tätigkeiten in der Forstwirtschaft (Bayern, Baden-Württemberg). Entsprechend geringer ist die Mehrfacharbeitslosigkeit in Nordrhein-Westfalen, wo beide Einflüsse unterdurchschnittlich wirken.

- Arbeitslose in Berufen mit geringer Qualifikation haben eine deutlich überdurchschnittliche Mehrfacharbeitslosigkeit. Aber auch Arbeitslose in qualifizierten Fertigungsberufen liegen mit einer Mehrfacharbeitslosigkeit von 1,82 noch deutlich über dem Durchschnitt. (Dies ist auch in der Österreich-Studie aufgefallen; siehe Fußnote 24.) Erst bei den qualifizierten Dienstleistungsberufen, in denen fast ausschließlich Angestellte arbeiten, ist die Mehrfacharbeitslosigkeit mit 1,23 deutlich geringer. Dieses Ergebnis wird noch durch den Berufsbereich IV - technische Berufe -mit Ingenieuren, Chemikern, Physikern, Mathematikern, Technikern und Technischen Sonderfachkräften, also einem Bereich mit durchweg hochqualifizierten Arbeitskräften, bestätigt. Hier liegt die Mehrfacharbeitslosigkeit auch mit Abstand am niedrigsten (Mehrfacharbeitslosigkeit 1,21). Insoweit können andere Befunde, die die Qualifikation als mitbestimmendes Merkmal nennen, erhärtet werden. (Die zuletzt genannte Gruppe ist zudem durch eine vergleichsweise günstige Beschäftigungslage, die sicherlich auch zu berücksichtigen ist, bevorzugt.) Auch in früheren IAB-Untersuchungen wurde der Einfluß unterschiedlicher Qualifikation auf die Mehrfacharbeitslosigkeit hervorgehoben ${ }^{26}$ ).

- Die Definition von 5er-Altersgruppen in einem Fünfjahres-Zeitraum ist nicht unproblematisch. Allerdings nur dort, wo Heterogenitäten bei bestimmten Altersgrenzen zu erwarten sind: Also am Anfang und am Ende der Skala. Fast alle Personen überschreiten im Beobachtungszeitraum eine der vorgegebenen Altersgrenzen. Im Kernbereich zwischen $25 / 30$ bis etwa 50 Jahren ist dies sicher unproblematisch. Ansonsten spielen Eingliederungs- und Ausgliederungsprobleme eine nicht kalkulierbare Rolle.

Beschränkt man sich bei der Betrachtung der Mehrfacharbeitslosigkeit zunächst auf den Kernbereich von 25 bis 50 Jahren, so sieht man in der Tat einige Unterschiede, die aber eher erratisch als systematisch wirken und keinesfalls den Schluß zulassen, Mehrfacharbeitslosigkeit sei bei Jüngeren häufiger anzutreffen als bei Älteren. Insoweit steht auch dieser Befund mehr im Einklang als im Widerspruch zu anderen Ergebnissen. Die deutlich rückläufige Mehrfacharbeitslosigkeit bei den über 50jährigen $(1,65$ und 1,20) 
muß jedenfalls sehr viel zurückhaltender interpretiert werden, wenn man bedenkt, daß die Höhe der Mehrfacharbeitslosigkeit in einem gegebenen Zeitraum nicht unabhängig ist von der Länge einzelner Arbeitslosigkeitsperioden; je länger diese sind, um so geringer muß die Zahl der Mehrfacharbeitslosigkeit ceteris paribus ausfallen. Insoweit wird die oben gebrauchte Formulierung nochmals aufgegriffen, daß der Einfluß des Alters auf den Umfang der Mehrfacharbeitslosigkeit eher nachrangig ist.

\subsubsection{Kumulative Arbeitslosigkeit}

Eine weit verbreitete Hypothese besagt, daß häufige Arbeitslosigkeitsperioden bei bestimmten Personengrup- pen mit überwiegend kürzeren Dauern verbunden sind, und daß umgekehrt geringere Mehrfacharbeitslosigkeit mit eher längeren Perioden einhergeht. Die Ergebnisse in Tabelle 1 deuten zunächst in diese Richtung.

- Als Beispiel sei einmal das Merkmal Geschlecht herausgegriffen: Die Männer haben eine Mehrfacharbeitslosigkeit von 1,86 bei einer durchschnittlichen Dauer pro Fall von 21,4 Wochen. Dies ergibt eine durchschnittliche Gesamtdauer von 39,9 Wochen pro Person im Beobachtungszeitraum. Dieser Wert wird von den Frauen mit 41,1 Wochen noch leicht überschritten, allerdings mit einer anderen Konstellation von Mehrfacharbeitslosigkeit und durchschnittlicher Dauer pro Fall: nämlich 1,52 und 27,1

Tabelle 1: Kennzahlen zur Mehrfacharbeitslosigkeit Juli 1979 - Juni 1984

\begin{tabular}{|c|c|c|c|c|c|}
\hline Merkmal & $\begin{array}{l}\text { Personen } \\
\text { (Mio.) }\end{array}$ & $\begin{array}{c}\text { Fälle } \\
\text { (Mio.) }\end{array}$ & $\varnothing \mathrm{MA}$ & $\begin{array}{c}\varnothing \text { Dauer } \\
\text { pro Person }\end{array}$ & $\begin{array}{l}\varnothing \text { Dauer } \\
\text { pro Fall }\end{array}$ \\
\hline \multicolumn{6}{|l|}{ Geschlecht } \\
\hline Männer & 5506 & 10268 & 1,86 & 39,9 & 21,4 \\
\hline Frauen & 4547 & 6891 & 1,52 & 41,1 & 27,1 \\
\hline \multicolumn{6}{|l|}{ Berufsbereiche } \\
\hline Pflanzenbauer & 198 & 421 & 2,13 & 40,2 & 18,9 \\
\hline Bergleute & 48 & 89 & 1,85 & 37,8 & 20,4 \\
\hline Fertigungsberufe & 4505 & 7896 & 1,75 & 40,0 & 22,8 \\
\hline Technische Berufe & 387 & 469 & 1,21 & 33,6 & 27,7 \\
\hline Dienstleistungsberufe & 4915 & 8284 & 1,69 & 39,3 & 23,3 \\
\hline \multicolumn{6}{|l|}{ Qualifikation*) } \\
\hline \multicolumn{6}{|l|}{ gering qualifizierte } \\
\hline Fertigungsberufe & 839 & 1606 & 1,91 & 48,5 & 25,3 \\
\hline Dienstleistungsberufe & 1253 & 2755 & 2,20 & 51,8 & 23,5 \\
\hline \multicolumn{6}{|l|}{ qualifizierte } \\
\hline Fertigungsberufe & 1355 & 2464 & 1,82 & 32,4 & 17,8 \\
\hline Dienstleistungsberufe & 2022 & 2496 & 1,23 & 33,7 & 27,3 \\
\hline \multicolumn{6}{|l|}{ Regionen } \\
\hline Schleswig-Holstein - Hamburg & 759 & 1370 & 1,81 & 41,3 & 22,9 \\
\hline Niedersachsen - Bremen & 1508 & 2480 & 1,64 & 42,3 & 25,7 \\
\hline Nordrhein-Westfalen & 3112 & 4667 & 1,50 & 41,2 & 27,5 \\
\hline Hessen & 778 & 1391 & 1,79 & 38,8 & 21,7 \\
\hline Rheinland-Pfalz - Saarland & 763 & 1344 & 1,76 & 41,2 & 23,4 \\
\hline Baden-Württemberg & 1138 & 2077 & 1,83 & 34,9 & 19,1 \\
\hline Nordbayern & 808 & 1470 & 1,82 & 41,8 & 23,0 \\
\hline Südbayern & 834 & 1668 & 2,00 & 39,4 & 19,7 \\
\hline Berlin (West) & 353 & 692 & 1,96 & 42,7 & 21,8 \\
\hline \multicolumn{6}{|l|}{ Altersgruppen } \\
\hline bis 19 Jahre & 1965 & 2240 & 1,14 & 20,4 & 17,9 \\
\hline $20-24$ Jahre & 2260 & 4205 & 1,86 & 34,2 & 18,4 \\
\hline 25 - 29 Jahre & 1440 & 2826 & 1,96 & 43,6 & 22,2 \\
\hline $30-34$ Jahre & 1016 & 1895 & 1,87 & 45,1 & 24,2 \\
\hline 35 - 39 Jahre & 784 & 1545 & 1,97 & 45,3 & 23,0 \\
\hline 40 - 44 Jahre & 771 & 1483 & 1,92 & 46,7 & 24,3 \\
\hline 45 - 49 Jahre & 627 & 1125 & 1,79 & 49,5 & 27,6 \\
\hline $50-54$ Jahre & 525 & 866 & 1,65 & 53,6 & 32,5 \\
\hline 55 - 59 Jahre & 665 & 795 & 1,20 & 55,8 & 46,7 \\
\hline $60-64$ Jahre & - & (179) & - & - & $(69,9)$ \\
\hline 65 Jahre und älter & - & - & - & - & - \\
\hline Insgesamt & 10053 & 17159 & 1,71 & 40,5 & 23,7 \\
\hline
\end{tabular}

*) Berufsordnungen (3-Steller), in denen der Anteil der Qualifizierten unter 30\% liegt, wurden für Fertigungs- und Dienstleistungsberufe getrennt zu „gering qualifizierten Berufen“ zusammengefaßt. Bei „qualifizierten Berufen“ betragen die Anteile der Ausgebildeten mindestens $85 \%$. 
Wochen. Bei den Frauen ist also die Mehrfacharbeitslosigkeit deutlich niedriger, die Gesamtbelastung durch Arbeitslosigkeit aber sogar leicht höher. (Auch die DIWStudie hebt dieses Ergebnis hervor.) ${ }^{27}$ )

- Ganz ähnlich liegt der Sachverhalt in einigen Berufsbereichen: Hohe Mehrfacharbeitslosigkeit bei den saisonabhängigen Berufen der Land- und Forstwirtschaft mit einer vergleichsweise geringen Dauer pro Fall führt auch hier zu einer Gesamtbelastung von 40,2 Wochen. Fertigungs- und Dienstleistungsberufe haben eine kumulierte Arbeitslosigkeit von durchschnittlich 40,0 bzw. 39,3 Wochen; dabei ist die Mehrfacharbeitslosigkeit bei den Fertigungsberufen mit einem hohen Arbeiteranteil mit 1,75 leicht höher als die der Angestellten in den Dienstleistungen mit 1,69.

- Die beachtlichen Unterschiede in der Mehrfacharbeitslosigkeit zwischen qualifizierten und gering qualifizierten Berufen lösen sich bei der kumulierten Arbeitslosigkeit zumindest innerhalb der Gruppen auf: Die Arbeitslosen in den gering qualifizierten Berufen haben eine kumulierte Dauer von 48,5 Wochen (Fertigungsberufe) bzw. 51,8 Wochen (Dienstleistungsberufe). Mit Abstand geringer belastet und im Niveau etwa gleich sind Arbeitslose in den qualifizierten Berufen. Sie haben eine kumulierte Dauer von 32,4 Wochen (Fertigungsberufe) bzw. 33,7 Wochen (Dienstleistungsberufe).

- Da die Mehrfacharbeitslosigkeit in den hier gewählten 5er-Altersgruppen dem erwarteten Muster offensichtlich nicht folgt, ist zu erwarten, daß die kumulierte Arbeitslosigkeit mit dem Alter deutlich zunimmt. Die Werte in Tabelle 1 bestätigen diese Annahme völlig: Die kumulierte Arbeitslosigkeit nimmt mit zunehmendem Alter von 34,2 Wochen bei den 20- bis 24jährigen auf 55,8 Wochen bei den 55- bis 59jährigen zu. Die älteren Arbeitslosen haben also nicht nur die lange Arbeitslosigkeitsperiode im Einzelfall, sondern über die vergleichsweise hohe Mehrfacharbeitslosigkeit auch die höchste kumulative Arbeitslosigkeit zu tragen.

- Es ist oben schon erwähnt worden, daß die Mehrfacharbeitslosigkeit in regionaler Hinsicht wesentlich durch saisonale Gegebenheiten geprägt wird; die regionale Arbeitsmarktsituation kommt damit in dieser Größe kaum zum Ausdruck (Mehrfacharbeitslosigkeit in Baden-Württemberg 1,83, in Nordrhein-Westfalen 1,50). Betrachtet man dagegen die kumulative Arbeitslosigkeit, so liegen BadenWürttemberg mit Abstand, Hessen und Südbayern immerhin noch geringfügig unter dem Durchschnitt.

Damit läßt sich resümierend festhalten, daß die kumulative Arbeitslosigkeit Problemlagen am Arbeitsmarkt am ehesten beschreibt: Sie liegt für den Beobachtungszeitraum bei rd. 40 Wochen; die meisten separat gemessenen Gruppen streuen nur geringfügig um diesen Wert. Sie zeigt aber deutlich günstigere und ungünstigere Arbeitsmarktsituationen für Personen und Regionen: Sie liegt vergleichsweise niedrig bei den Berufen hoher Qualifikation (ca. 33 Wochen), sie liegt vergleichsweise niedrig in Baden-Württemberg (34,9 Wochen); sie steigt mit zunehmendem Alter (bis 55,8 Wochen) und sie zeigt die hohe Belastung durch Arbeitslosigkeit in den gering qualifizierten Tätigkeiten (ca. 50 Wochen).

${ }^{27}$ ) Schupp, J., Dynamische Aspekte bei der Beobachtung von Arbeitslosigkeit, a.a.O., S. 17

\subsubsection{Mehrfacharbeitslosigkeit versus kumulative Arbeitslosigkeit}

Die Mehrfacharbeitslosigkeit als zusätzliche Risikokomponente (zu Zugangs- und Dauerrisiko) ist schon seit einiger Zeit innerhalb der dynamischen Betrachtung des Arbeitsmarktes in der Diskussion. Im Zusammenhang damit erschien auch die kumulative Arbeitslosigkeit bzw. die „Gesamtbelastung“ durch Arbeitslosigkeit. Aber nach wie vor steht die Mehrfacharbeitslosigkeit im Vordergrund der Diskussion. Die hier vorgestellten Ergebnisse geben immerhin Anlaß zu überlegen, ob nicht die kumulative Arbeitslosigkeit das Problem instabiler Erwerbsbiographien besser beschreibt. Dafür sprechen schon methodische Gründe: Die häufig bereits durch die Datenlage notwendige enge Begrenzung des Beobachtungszeitraumes ergibt bei kurzen Dauern häufigere Mehrfacharbeitslosigkeit als bei langen, was in isolierter Betrachtung ein unzutreffendes Bild zeichnet. Auch gibt es eine Vielzahl von Ereignissen, die die Arbeitslosigkeit oft nur „technisch“ unterbrechen und insoweit zu Mehrfacharbeitslosigkeit führen, ohne daß die Arbeitslosigkeit für den Betroffenen damit zu Ende wäre. Dies reicht von Unterbrechung durch Krankheit (in dieser Untersuchung allerdings ausgeschlossen) bis zu den unterschiedlichsten Maßnahmen, z.B. Arbeitsbeschaffungsmaßnahmen .

Aber auch Unterbrechungen durch kurzfristige Beschäftigungen, die zu hoher Mehrfacharbeitslosigkeit bei kurzen Dauern führen, werden von den Betroffenen sicher nicht als erlösende Reintegration empfunden; die kumulative Dauer zeigte auch eine fast verblüffend hohe Übereinstimmung bei den meisten betrachteten Gruppen. Da die Mehrfacharbeitslosigkeit mit der Dauer pro Fall mit $\mathrm{r}=$ - 0,54 deutlich negativ korreliert ist, ist die kumulative Arbeitslosigkeit zwangsläufig gleichmäßiger verteilt und läßt die wenigen noch vorhandenen Unterschiede leichter interpretieren. Insoweit liegt es nahe, das Problem instabiler oder instabil gewordener Erwerbsverläufe von vornherein mit kumulativer Arbeitslosigkeit (mit-)zubeschreiben.

\subsection{Entwicklung von Mehrfacharbeitslosigkeit und kumulativer Arbeitslosigkeit}

Bei der Kommentierung der oben vorgestellten Ergebnisse anderer Autoren wurde schon darauf hingewiesen, daß praktisch alle Studien in wesentlichen, die Anlage der Untersuchungen betreffenden Aspekten nicht übereinstimmen. Dazu gehören insbesondere die Länge und Lage des Beobachtungszeitraumes. Auf den Einfluß dieser Größen auf die Ergebnisse wird generell zuwenig hingewiesen. Viele Arbeiten lesen sich so, als sei Mehrfacharbeitslosigkeit eine Art „Naturkonstante“, die nun - weil die Datenlage jetzt gerade günstig ist - eben im vorgegebenen Zeitraum ermittelt wurde.

Es wurde im Abschnitt über offene Methodenfragen bereits gezeigt, daß Mehrfacharbeitslosigkeit in einem EinJahreszeitraum anders in Erscheinung tritt als in einem Fünf-Jahreszeitraum. Da angenommen werden kann, daß Mehrfacharbeitslosigkeit nicht nur eine zeitraumabhängige, sondern bei festgelegten Zeiträumen auch eine zeitabhängige Größe ist, sind Vergleiche zwischen früheren und neueren Untersuchungen zur Mehrfacharbeitslosigkeit noch viel problematischer. Will man nämlich wissen, ob Mehrfacharbeitslosigkeit bzw. die Konzentration von Arbeitslosigkeit bei bestimmten Personengruppen größer wird oder sich sonstwie verändert, müßte man im Grunde das gleiche Untersuchungsdesign in verschiedenen Zeitab- 
Tabelle 2: Mehrfacharbeitslosigkeit in Ein-Jahreszeiträumen (Fälle und Personen in 1000; Dauer in Wochen)

\begin{tabular}{|c|c|c|c|c|c|c|c|c|c|c|}
\hline & 1977 & 1978 & 1979 & 1980 & 1981 & 1982 & 1983 & 1984 & 1985 & 1986 \\
\hline Fälle & 2324 & 2186 & 1923 & 1964 & 2363 & 2600 & 2885 & 2744 & 2749 & 2666 \\
\hline Personen & 1955 & 1841 & 1647 & 1645 & 1961 & 2196 & 2439 & 2339 & 2333 & 2276 \\
\hline $\begin{array}{l}\text { Mehrfach- } \\
\text { arbeitslosigkeit }\end{array}$ & 1,19 & 1,19 & 1,17 & 1,19 & 1,20 & 1,18 & 1,18 & 1,17 & 1,18 & 1,17 \\
\hline Dauer pro Fall & 16,4 & 16,7 & 16,4 & 15,4 & 16,1 & 19,2 & 22,3 & 24,1 & 24,9 & 26,6 \\
\hline Dauer pro Person & 19,5 & 19,9 & 19,1 & 18,4 & 19,4 & 22,7 & 26,4 & 28,3 & 29,4 & 31,2 \\
\hline
\end{tabular}

schnitten einsetzen, um den Einfluß unterschiedlich langer Zeiträume von der Zeit selbst trennen zu können. Solche Untersuchungen liegen unseres Wissens nach nicht vor. Eher besteht die Neigung, Ergebnisse aus den siebziger Jahren mit denen der Gegenwart zu vergleichen.

Es ist ohne Frage auch sehr schwierig, Untersuchungen zur Mehrfacharbeitslosigkeit so zu konzipieren, daß sie in fünf oder zehn Jahren in einer heute überhaupt nicht abschätzbaren Arbeitsmarktsituation genau in der gleichen Weise wiederholt werden können.

Um trotzdem Hinweise auf die Entwicklung (nicht mehr auf das Niveau, das im vorigen Abschnitt beschrieben wurde) von Mehrfacharbeitslosigkeit und kumulativer Arbeitslosigkeit geben zu können, wurde die Datei der Leistungsempfänger in gleitenden Zeiträumen von 1977 bis 1986 ausgewertet. Die mit den Daten über Leistungsbezugszeiten als Ersatz für Zeiten der Arbeitslosigkeit verbundenen Probleme wurden eingangs bereits erörtert; daß das Niveau von Mehrfacharbeitslosigkeit systematisch untererfaßt wird, wurde mehrfach betont. Andererseits wird Arbeitslosigkeit in ihrem Prozeßcharakter formal durch Leistungsfälle, leistungsberechtigte Personen, Dauer des Leistungsbezugs und Häufigkeit des Leistungsbezugs adäquat abgebildet, so daß für die hier angeschnittene zeitliche Entwicklung der Mehrfacharbeitslosigkeit zumindest die Tendenzen aus der Leistungsempfänger-Datei ermittelt werden können.

Um unhandliche Begriffe wie mehrfacher Leistungsbezug, Dauer des Leistungsbezuges usw. zu vermeiden, ist auch hier von Mehrfacharbeitslosigkeit und kumulativer Arbeitslosigkeit die Rede.

\section{2. l Die globale Entwicklung der Mehrfacharbeitslosigkeit in Fünf-Jahres-Zeiträumen}

Tabelle 3 zeigt für den Zeitraum 1/1977 bis 12/1981, also für 5 Jahre, eine Mehrfacharbeitslosigkeit von 1,68. Die durchschnittliche Dauer pro Periode betrug 16,2 Wochen. Verschiebt man diesen Zeitraum gleitend um jeweils ein Jahr, so sieht man, daß sich sowohl die durchschnittliche Periodendauer (auf 23,4 Wochen) als auch die durchschnittliche Mehrfacharbeitslosigkeit (auf 1,76) kontinuierlich erhöht. Der gleichzeitige Anstieg beider Größen ist insoweit beachtlich, als bei dieser Konstellation eine Überschätzung der Mehrfacharbeitslosigkeit mit Sicherheit ausgeschlossen werden kann. (Würde umgekehrt ein Anstieg der Mehrfacharbeitslosigkeit mit einem Rückgang der durchschnittlichen Periodenlänge einhergehen, müßte immerhin in Erwägung gezogen werden, daß die Veränderung der Relation Zeitraum/Periodenlänge das Ergebnis mit beeinflußt.)

Aus der ansteigenden Periodendauer und ansteigender Mehrfacharbeitslosigkeit ergibt sich natürlich auch ein Anstieg der kumulierten Dauer pro Person, und zwar von 27,2 Wochen auf 41,1 Wochen. Die globale Tendenz kann damit so interpretiert werden: In einem Zeitraum gegebener Länge (hier: 5 Jahre) nehmen im Zeitverlauf sowohl die Arbeitslosigkeitsperioden pro Person wie auch die Dauer pro Periode zu. Die Mehrfacharbeitslosigkeit ,verdichtet" sich oder die Zeiträume zwischen wiederholter Arbeitslosigkeit werden kürzer.

Die Aussage von der Strukturalisierung der Arbeitslosigkeit, die die Selektionsmechanismen beim Eintritt des

Tabelle 3: Mehrfacharbeitslosigkeit in gleitenden Fünf-Jahres-Zeiträumen (Fälle und Personen in 1000; Dauer in Wochen)

\begin{tabular}{|c|c|c|c|c|c|c|}
\hline & $1977-81$ & $1978-82$ & $1979-83$ & $1980-84$ & $1981-85$ & $1982-86$ \\
\hline Fälle & 10760 & 11037 & 11736 & 12557 & 13341 & 13644 \\
\hline Personen & 6395 & 6545 & 6901 & 7267 & 7613 & 7759 \\
\hline \multicolumn{7}{|l|}{ Mehrfach- } \\
\hline arbeitslosigkeit & 1,68 & 1,69 & 1,70 & 1,73 & 1,75 & 1,76 \\
\hline Dauer pro Fall & 16,2 & 16,8 & 18,2 & 19,8 & 21,5 & 23,4 \\
\hline Dauer pro Person & 27,2 & 28,4 & 30,9 & 34,1 & 37,6 & 41,1 \\
\hline
\end{tabular}

Tabelle 4: Mehrfacharbeitslosigkeit in sukzessiv erweiterten Zeiträumen (Fälle und Personen in 1000; Dauer in Wochen)

\begin{tabular}{|c|c|c|c|c|c|c|c|c|c|c|}
\hline & $1977-77$ & $1977-78$ & $1977-79$ & $1977-80$ & $1977-81$ & $1977-82$ & $1977-83$ & $1977-84$ & $1977-85$ & $1977-86$ \\
\hline Fälle & 2323 & 4510 & 6432 & 8396 & 10759 & 13358 & 16242 & 18984 & 21731 & 24395 \\
\hline Personen & 1955 & 3270 & 4278 & 5256 & 6395 & 7621 & 8937 & 10122 & 11238 & 12291 \\
\hline $\begin{array}{l}\text { Mehrfach- } \\
\text { arbeitslosigkeit }\end{array}$ & 1,19 & 1,38 & 1,50 & 1,60 & 1,68 & 1,75 & 1,82 & 1,88 & 1,93 & 1,98 \\
\hline Dauer pro Fall & 16,4 & 16,6 & 16,5 & 16,2 & 16,2 & 16,8 & 17,8 & 18,7 & 19,5 & 20,3 \\
\hline Dauer pro Person & 19,5 & 22,8 & 24,8 & 26,0 & 27,3 & 29,4 & 32,3 & 35,1 & 37,7 & 40,2 \\
\hline
\end{tabular}


Ereignisses Arbeitslosigkeit und bei der Wiedereinstellung nach unterschiedlichen Verweildauern zusammenfaßt, ist nach diesem Befund um eine weitere Komponente zu ergänzen: Die Strukturalisierung findet nicht nur in der Weise statt, daß sie im Laufe der Zeit immer mehr „Problemfälle“ immer länger im Arbeitslosenbestand beläßt, sondern auch in der Weise, daß immer häufiger immer wieder die gleichen Personen von Arbeitslosigkeit betroffen werden. Und dies ist nicht etwa eine Folge veränderter Arbeitslosigkeit oder sich ändernder Umschlagsprozesse: Der Anstieg der Mehrfacharbeitslosigkeit erfolgt bei seit Jahren konstantem Arbeitslosigkeitsniveau von jahresdurchschnittlich über 2 Millionen und vergleichsweise konstanten $\mathrm{Zu}$ - und Abgängen von jährlich etwa 3,7 Millionen. Insoweit kann es sich nur um eine Verstärkung der Konzentration von Arbeitslosigkeit auf bestimmte Personen oder Personengruppen handeln.

\subsubsection{Die Verteilung der Arbeitslosigkeitsperioden in der zeitlichen Entwicklung}

Aus der Tabelle 5 kann man ersehen, wie sich die Arbeitslosigkeitsfälle (die in der ganz kurzen Frist identisch sind mit arbeitslosen Personen) im Laufe von 10 Jahren zur Häufung bei zwei, drei oder noch mehr Arbeitslosigkeitsperioden pro Person hin entwickeln. Das Ausgangsjahr 1977 restringiert durch seine relative Kürze das Auftreten von Mehrfacharbeitslosigkeit beträchtlich: $84,1 \%$ der arbeitslosen Personen haben genau eine Arbeitslosigkeitsperiode. „Nur“ $13,5 \%$ erleben 2 Perioden und 2,4\% drei und mehr. Im Jahre 1978 kommen (bei nahezu gleichem Volumen der Arbeitslosigkeit, s. Tabelle 7) weitere 1,315 Mio. arbeitslose Personen hinzu (Differenz aus Spalte 1 und 2). Die Entwicklung in den folgenden Jahren zeigt eine weitere Verschiebung zu den Personen mit jeweils zwei Perioden, parallel dazu bei jenen mit drei Perioden und bei Arbeitslosen mit mehr als 4 Perioden.
Bildet man zwischen den Spalten der Tabelle 5 die Differenzen, erhält man die jährlichen Veränderungen. Man schaltet damit den Zeitraumeffekt der Tabelle 5 (1977-1977, 1977-1978 usw.) aus. Die Ergebnisse enthält Tabelle 6. Sie darf allerdings nicht fehlinterpretiert werden. Die Summenzeile enthält die jährlich neu hinzukommenden arbeitslosen Personen, die im vorangegangenen Zeitraum (bzw. bis dahin) noch nicht mit Arbeitslosigkeit in Erscheinung getreten sind. Die Aufteilung auf Perioden ist dagegen nur zahlenmäßig identisch, nicht aber in den Personen. Dazu ein Beispiel: Die Differenzen zwischen den Zeiträumen $1 / 1977$ bis $12 / 1977$ und $1 / 1977$ bis $12 / 1978$ in Tabelle 5 ergeben die Veränderungen durch das Jahr 1978 in Tabelle 6. Sie besagen, daß 1,315 Mio. zusätzliche arbeitslose Personen zu den 1,955 Mio. des Jahres 1977 hinzugekommen sind. Die Aufteilung auf Perioden darf aber nicht so interpretiert werden, als ob von den 1,315 Mio. zusätzlichen Arbeitslosen genau 692.000 eine Periode, 444.000 zwei Perioden usw. erfahren mußten. Diese 444.000 Arbeitslosen mit genau zwei Perioden verteilen sich sowohl auf neu hinzugekommene Arbeitslose wie auch auf jene des Jahres 1977, die dort nur eine Periode aufwiesen, in 1978 aber erneut arbeitslos wurden. Der Vorteil der Differenzenbildung in Tabelle 6 gegenüber den gleitenden Summen in Tabelle 5 liegt an der stärkeren Isolierung des Konzentrationsvorganges. Tabelle 6 zeigt viel deutlicher, wie sich die Mehrfacharbeitslosigkeit zunächst zu den Personen mit jeweils zwei Perioden, noch länger ansteigend bei jenen mit drei Perioden und am stärksten und längsten ansteigend bei denen mit vier und mehr als vier Perioden hin entwickelt.

Im Zeitraum von $1 / 1977$ bis $12 / 1986$ sind also insgesamt 12,292 Mio. verschiedene Personen ein oder mehrmals arbeitslos geworden.

Stellt man dieser Personenbetrachtung noch in gleicher Weise die Volumenbetrachtung zur Seite $($ Volumen $=$

Tabelle 5: Entwicklung der Mehrfacharbeitslosigkeit im zeitlichen Verlauf (Personen)

Die arbeitslosen Personen* im Zeitraum ... verteilen sich auf ... Perioden

\begin{tabular}{|c|c|c|c|c|c|c|c|c|c|c|}
\hline Perioden $\begin{array}{r}\text { Zeitraum } \\
\text { Jahre }\end{array}$ & $1977-77$ & $1977-78$ & $1977-79$ & $1977-80$ & $1977-81$ & $1977-82$ & $1977-83$ & $1977-84$ & $1977-85$ & $1977-86$ \\
\hline & \multicolumn{10}{|c|}{ in Tsd. } \\
\hline 1 & 1644 & 2336 & 2845 & 3355 & 3940 & 4548 & 5162 & 5703 & 6195 & 6665 \\
\hline 2 & 264 & 708 & 928 & 1148 & 1411 & 1709 & 2043 & 2322 & 2573 & 2803 \\
\hline 3 & 40 & 171 & 358 & 441 & 564 & 708 & 877 & 1036 & 1180 & 1307 \\
\hline 4 & 6 & 42 & 102 & 205 & 253 & 322 & 408 & 494 & 583 & 664 \\
\hline Rest & 1 & 13 & 45 & 106 & 227 & 334 & 447 & 567 & 707 & 853 \\
\hline Summe & 1955 & 3270 & 4278 & 5255 & 6395 & 7621 & 8937 & 10122 & 11238 & 12292 \\
\hline \multirow[t]{2}{*}{$\begin{array}{l}\text { Veränderung } \\
\text { zum Vorjahr }\end{array}$} & - & 1315 & 1008 & 977 & 1140 & 1226 & 1316 & 1185 & 1116 & 1054 \\
\hline & \multicolumn{10}{|c|}{ in $\%$} \\
\hline 1 & 84,1 & 71,4 & 66,5 & 63,8 & 61,6 & 59,7 & 57,8 & 56,3 & 55,1 & 54,2 \\
\hline 2 & 13,5 & 21,7 & 21,7 & 21,8 & 22,1 & 22,4 & 22,9 & 22,9 & 22,9 & 22,8 \\
\hline 3 & 2,0 & 5,2 & 8,4 & 8,4 & 8,8 & 9,3 & 9,8 & 10,2 & 10,5 & 10,6 \\
\hline 4 & 0,3 & 1,3 & 2,4 & 3,9 & 4,0 & 4,2 & 4,6 & 4,9 & 5,2 & 5,4 \\
\hline Rest & 0,1 & 0,4 & 1,1 & 2,0 & 3,5 & 4,4 & 5,0 & 5,6 & 6,3 & 6,9 \\
\hline Summe & 100,0 & 100,0 & 100,0 & 100,0 & 100,0 & 100,0 & 100,0 & 100,0 & 100,0 & 100,0 \\
\hline
\end{tabular}

*) Personen $=$ Leistungsempfänger 
Tabelle 6: Entwicklung der Mehrfacharbeitslosigkeit im zeitlichen Verlauf (Personen) Zusätzliche Personen im Jahr ... und Veränderung zwischen den Perioden

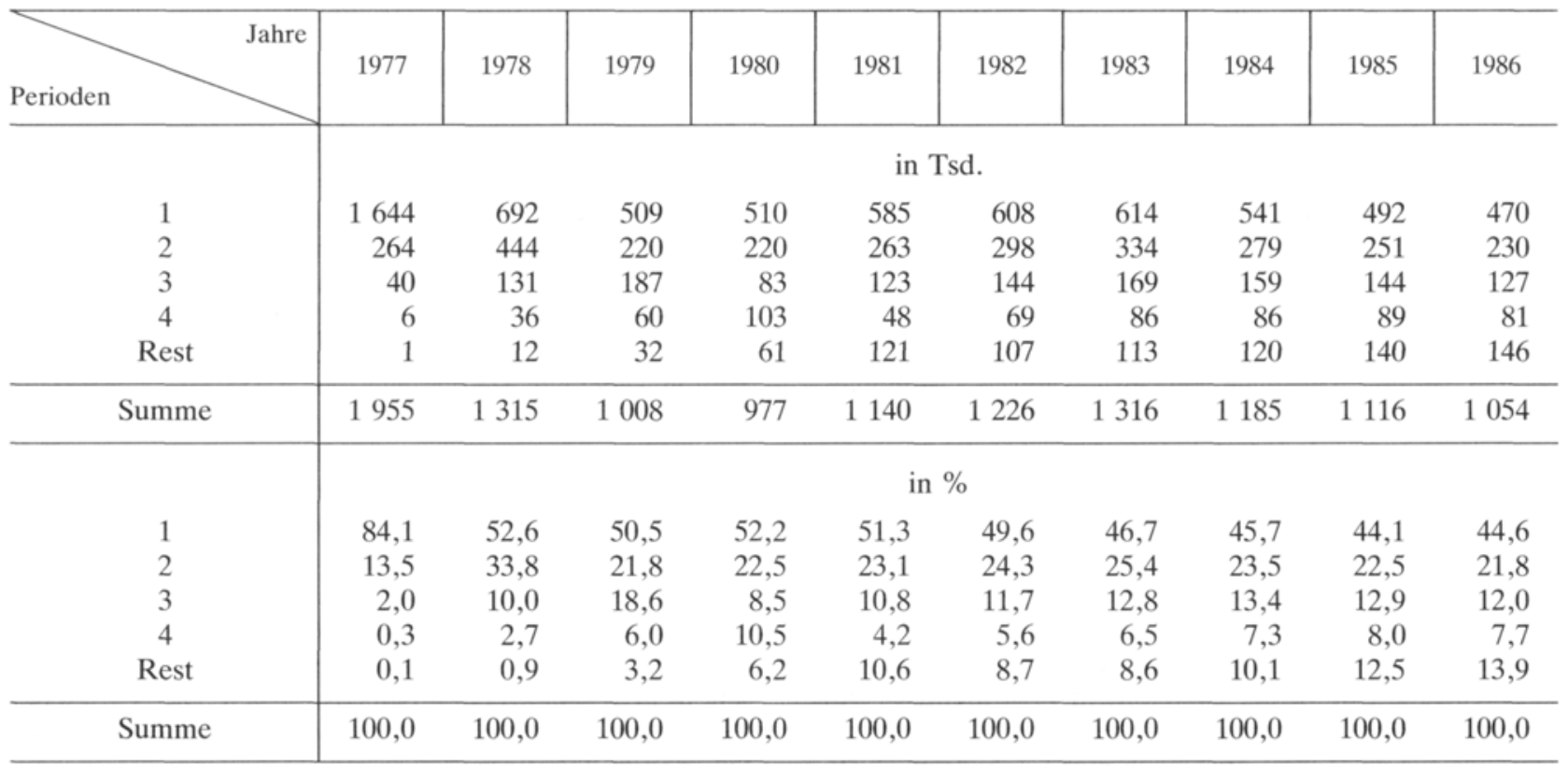

*) Personen $=$ Leistungsempfänger

Tabelle 7: Entwicklung der Mehrfacharbeitslosigkeit im zeitlichen Verlauf (Volumen)

Das (Leistungs-) Volumen im Zeitraum ... verteilt sich auf ... Perioden

\begin{tabular}{|c|c|c|c|c|c|c|c|c|c|c|}
\hline $\begin{array}{r}\begin{array}{r}\text { Zeitraum } \\
\text { Jahre }\end{array} \\
\text { Perioden }\end{array}$ & $1977-77$ & $1977-78$ & $1977-79$ & $1977-80$ & $1977-81$ & $1977-82$ & $1977-83$ & $1977-84$ & $1977-85$ & $1977-86$ \\
\hline & \multicolumn{10}{|c|}{ in Tsd. } \\
\hline 1 & 708 & 1106 & 1368 & 1588 & 1862 & 2243 & 2713 & 3159 & 3570 & 3974 \\
\hline 2 & 123 & 414 & 607 & 780 & 988 & 1262 & 1624 & 1970 & 2306 & 2628 \\
\hline 3 & 20 & 115 & 279 & 373 & 506 & 680 & 911 & 1157 & 1408 & 1659 \\
\hline 4 & 3 & 30 & 88 & 204 & 271 & 374 & 516 & 677 & 859 & 1047 \\
\hline Rest & 2 & 11 & 41 & 115 & 287 & 474 & 716 & 1002 & 1359 & 1787 \\
\hline Summe & 856 & 1676 & 2383 & 3060 & 3914 & 5033 & 6480 & 7965 & 9502 & 11095 \\
\hline \multirow[t]{2}{*}{$\begin{array}{l}\text { Veränderung } \\
\text { zum Vorjahr }\end{array}$} & - & 820 & 707 & 677 & 854 & 1119 & 1447 & 1485 & 1537 & 1593 \\
\hline & \multicolumn{10}{|c|}{ in $\%$} \\
\hline 1 & 82,7 & 66,0 & 57,4 & 51,9 & 47,6 & 44,6 & 41,9 & 39,7 & 37,6 & 35,8 \\
\hline 2 & 14,4 & 24,7 & 25,5 & 25,5 & 25,2 & 25,1 & 25,1 & 24,7 & 24,3 & 23,7 \\
\hline 3 & 2,3 & 6,9 & 11,7 & 12,2 & 12,9 & 13,5 & 14,1 & 14,5 & 14,8 & 15,0 \\
\hline 4 & 0,4 & 1,8 & 3,7 & 6,7 & 6,9 & 7,4 & 8,0 & 8,5 & 9,0 & 9,4 \\
\hline Rest & 0,2 & 0,7 & 1,7 & 3,8 & 7,3 & 9,4 & 11,0 & 12,6 & 14,3 & 16,1 \\
\hline Summe & 100,0 & 100,0 & 100,0 & 100,0 & 100,0 & 100,0 & 100,0 & 100,0 & 100,0 & 100,0 \\
\hline
\end{tabular}

*) Personen $=$ Leistungsempfänger

Summe aller Arbeitslosigkeitszeiten dieser Personen), Tabellen 7 und 8, so sieht man schnell den Kumulationseffekt der Mehrfacharbeitslosigkeit: Während die arbeitslosen Personen von 1,955 Mio. im Jahre 1977 auf 12,292 Mio. bis 1986 anwuchsen, also etwa um den Faktor 6,3, erhöhte sich das Volumen von 856.000 Personenjahren in 1977 auf 11,095 Mio. Personenjahre im Zeitraum 1977 bis 1986. Dies entspricht dem Faktor 13,0. Arbeitslosigkeit häuft sich damit bei den betroffenen Personen immer mehr an. Auch bei der Betrachtung der Volumengrößen (Tabellen 7 und 8) sieht man, wie sie sich von der in der kurzen Sicht dominierenden Einer-Periode schnell wegverlagern und wie sie - absolut und relativ - in den Kategorien mit zwei und mehr Perioden zunehmen. Auch hier wird nochmals deutlich, welche dominante Rolle die Länge des Beobachtungszeitraumes spielt. 
Tabelle 8: Entwicklung der Mehrfacharbeitslosigkeit im zeitlichen Verlauf (Volumen) Zusätzliches Volumen im Jahr ... und Veränderung zwischen den Perioden

\begin{tabular}{|c|c|c|c|c|c|c|c|c|c|c|}
\hline 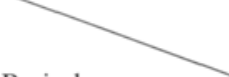 & 1977 & 1978 & 1979 & 1980 & 1981 & 1982 & 1983 & 1984 & 1985 & 1986 \\
\hline & \multicolumn{10}{|c|}{ in Tsd. } \\
\hline 1 & 708 & 398 & 262 & 220 & 274 & 381 & 470 & 446 & 411 & 404 \\
\hline 2 & 123 & 291 & 193 & 173 & 208 & 274 & 362 & 346 & 336 & 322 \\
\hline 3 & 20 & 95 & 164 & 94 & 133 & 174 & 231 & 246 & 251 & 251 \\
\hline 4 & 3 & 27 & 58 & 116 & 67 & 103 & 142 & 161 & 182 & 188 \\
\hline Rest & 2 & 9 & 30 & 74 & 172 & 187 & 242 & 286 & 357 & 428 \\
\hline \multirow[t]{2}{*}{ Summe } & 856 & 820 & 707 & 677 & 854 & 1119 & 1447 & 1485 & 1537 & 1593 \\
\hline & \multicolumn{10}{|c|}{ in $\%$} \\
\hline 1 & 82,7 & 48,5 & 37,1 & 32,5 & 32,1 & 34,0 & 32,5 & 30,0 & 26,7 & 25,4 \\
\hline 2 & 14,4 & 35,5 & 27,3 & 25,6 & 24,4 & 24,5 & 25,0 & 23,3 & 21,9 & 20,2 \\
\hline 3 & 2,3 & 11,6 & 23,2 & 13,9 & 15,6 & 15,5 & 16,0 & 16,6 & 16,3 & 15,8 \\
\hline 4 & 0,4 & 3,3 & 8,2 & 17,1 & 7,8 & 9,2 & 9,8 & 10,8 & 11,8 & 11,8 \\
\hline Rest & 0,2 & 1,1 & 4,2 & 10,9 & 20,1 & 16,7 & 16,7 & 19,3 & 23,2 & 26,9 \\
\hline Summe & 100,0 & 100,0 & 100,0 & 100,0 & 100,0 & 100,0 & 100,0 & 100,0 & 100,0 & 100,0 \\
\hline
\end{tabular}

*) Personen $=$ Leistungsempfänger

Verknüpft man die Personenbetrachtung mit der Volumenbetrachtung, so sieht man, daß beispielsweise im 10-Jahreszeitraum 1977 bis $198654,2 \%$ der betroffenen Personen „nur“ $35,8 \%$ des gesamten Arbeitslosigkeitsvolumens mit genau einer Arbeitslosigkeitsperiode $\mathrm{zu}$ tragen haben. Andererseits entfallen auf 6,9\% bzw. 853.000 Arbeitslose mit mehr als 4 Perioden 16,1\% des gesamten Arbeitslosigkeitsvolumens.

Es wird hier das gleiche Phänomen sichtbar, das auch bei längerfristiger Arbeitslosigkeit zu beobachten ist: Obwohl die davon Betroffenen von der absoluten Größenordnung her die Arbeitslosenzahl nicht dominieren, haben sie allein über die Länge ihrer Arbeitslosigkeitsperioden einen weit überproportionalen Anteil der Gesamtarbeitslosigkeit zu tragen. Allerdings sind die Personen bzw. Personengruppen, bei denen längerfristige Arbeitslosigkeit auftritt, bekannt, so daß arbeitsmarktpolitische Hilfestellung gegeben werden kann.

Bei der hier beschriebenen und gemessenen Mehrfacharbeitslosigkeit - und der sich durch sie ergebenden kumulativen Arbeitslosigkeit - ist dies viel komplizierter: Viele von ihnen sind in der Gruppe jener mit nur kurzen Verweildauern in der Arbeitslosigkeit enthalten und - zumindest statistisch - nicht als ebenso problematische Fälle wie die Langfristarbeitslosen erkennbar.

\section{Ursachen von Mehrfacharbeitslosigkeit und kumulativer Arbeitslosigkeit}

\subsection{Erklärungsversuche}

Das Auftreten mehrfacher Arbeitslosigkeit wurde und wird zum Teil unterschiedlich begründet, wobei ganz sicher auch unterschiedliche Situationen, Verhaltensweisen, Einstellungen in verschiedenen Ländern eine Rolle spielen.

Der Arbeitsmarkt in Nordamerika (USA, Kanada) hat andere Charakteristika als Arbeitsmärkte in Westeuropa: Der ungleich höheren Fluktuation und Mobilität dort - aus vielfältigen Untersuchungen zum labour-turnover bekannt
- steht hierzulande ein eher stabiler, verfestigter, weniger beweglicher Arbeitsmarkt gegenüber.

- Ein Arbeitsmarkt mit mehr stabilen und längerfristigen Beschäftigungsverhältnissen (bedingt z.B. auch durch Kurzarbeit versus layoff).

- Ein Arbeitsmarkt mit geringeren Risiken, arbeitslos zu werden, aber auch - bei hoher und anhaltender Arbeitslosigkeit - mit höheren Verbleibsrisiken.

Mehrfacharbeitslosigkeit ist deshalb beispielsweise in den USA schon eine lange bekannte und häufig untersuchte Erscheinung. Ein großer Teil der dort beobachteten Mehrfacharbeitslosigkeit (mit in der Regel kurzer Dauer) wird dann auch den Jugendlichen (oder jüngeren) zugeschrieben, den Männern mittleren Alters, die in saisonabhängigen Betrieben der Landwirtschaft, des Baugewerbes und verschiedener Fertigungszweige beschäftigt sind, aber auch all jenen Personen, die bereits mehrere Arbeitslosigkeitsperioden hinter sich haben.

Solche Ergebnisse haben auch hierzulande zu der sehr vereinfachten Betrachtungsweise geführt, Mehrfacharbeitslosigkeit mit überwiegend kurzen Dauern sei vor allem bei jüngeren Arbeitnehmern oder Arbeitslosen anzutreffen, bei älteren eher seltener.

Soweit sich in ihnen nur Übergangsprobleme vom Bildungs- ins Beschäftigungssystem zeigen, die sich später auflösen, seien sie weniger gewichtig als auf Dauer konzentrierte und verfestigte Arbeitslosigkeit bei bestimmten Personengruppen. Das gleiche gilt für die sich wiederholende Saisonarbeitslosigkeit.

Es ist unschwer zu erkennen, daß die Arbeitsmarktexperten der OECD zunächst auch diese Hypothesen geprüft haben. Sie sind dabei aber für westeuropäische Staaten zu teilweise ganz anderen Ergebnissen gekommen und mußten andererseits bisherige Ergebnisse für die USA in Frage stellen: „In Danmark and Britain, persons who experience multiple spells in one year are more likely than other groups to experience multiple spells in the following year. 
This probability was similar for different demographic groups“ ${ }^{28}$ ) Für die USA wird dagegen festgestellt: „These data suggest that the persistence of multiple spells in the United States is less than in Danmark or Great Britain““. ${ }^{29}$ ) Allerdings wird dann auch unterschieden zwischen dem Ausmaß der höheren Mehrfacharbeitslosigkeit nach eingetretener Arbeitslosigkeit (s.o.) und der Entwicklungstendenz: „While the persistence of multiple spells over time varied among countries, there was a tendency in each of the three countries (Danmark, Great Britain, United States; d.V.) for which data were available for persons who experienced multiple spells in one year to be more likely to experience them in the next year". ${ }^{30}$ ) Die Gewichtung

- Saisonarbeitslosigkeit,

- hoher turnover bei Jüngeren,

- Destabilisierung/Segmentation

wurde qua Reihenfolge beibehalten, aber in ihrer Bedeutung - vor allem auch bei den Jugendlichen - doch anders interpretiert. Dieses Schema, das Arbeitsmarktexperten der Bundesrepublik Deutschland aufgrund von Diskussionen und Ergebnissen Ende der 70er Jahre ebenfalls geläufig ist, wurde inzwischen für die Bundesrepublik schon mehrfach in Frage gestellt. Dazu haben zwei Aspekte entscheidend beigetragen:

- Die früher übliche Betrachtung der Mehrfacharbeitslosigkeit und kumulativen Arbeitslosigkeit in kurzen Perioden, in denen beide Phänomene nicht vollständig und damit nicht richtig erfaßt werden konnten, wurde abgelöst durch eine Beobachtung in längeren Zeiträumen.

- In den 70er Jahren dominierte die von personenbezogenen Merkmalen geprägte Zugangsstruktur auch die Mehrfacharbeitslosigkeit, die sich mithin in ihr stark auswirkte.

Im folgenden wird versucht, aus den hier vorgestellten Berechnungen auch Anhaltspunkte für besondere Risikolagen, die Mehrfacharbeitslosigkeit und kumulative Arbeitslosigkeit begünstigen, zu finden.

\subsection{Ursachen für Mehrfacharbeitslosigkeit in der gegenwärtigen} Arbeitsmarktsituation der Bundesrepublik Deutschland

Wie oben schon angedeutet wurde, sind im Grunde drei Ursachenkomplexe gegeneinander abzugrenzen, näher zu spezifizieren und in ihrem Einfluß auf die Mehrfacharbeitslosigkeit zu gewichten:

- die wiederkehrende Saisonarbeitslosigkeit,

- die Mehrfacharbeitslosigkeit bei bestimmten demographischen Gruppen (z.B. den Jugendlichen),

- die Mehrfacharbeitslosigkeit im Anschluß an Arbeitslosigkeit (Destabilisierung).

\section{2. l Saisonarbeitslosigkeit}

Die Saisonarbeitslosigkeit macht ganz sicher einen Teil der hier festgestellten Mehrfacharbeitslosigkeit aus. In berufli-

\footnotetext{
$\left.{ }^{28}\right)$ OECD, a.a.O., S. 107

) ebenda, S. 107

${ }^{30}$ ) ebenda, S. 113

$\left.{ }^{31}\right)$ Karr, W., Aspekte saisonaler Arbeitslosigkeit, in: MittAB 1/83, S. 21

32) Brinkmann, C., Karr, W., Spitznagel, E., Saisonale Schwankungen von Beschäftigung und Arbeitslosigkeit, in: MittAB 4/85, S. 434

${ }^{33}$ ) IAB-Kurzbericht vom 29. 7. 1977, in: BeitrAB 42, Nürnberg 1979

${ }^{34}$ ) Brinkmann, C., Verbleib und Vermittlungsprobleme von Arbeitslosen, in: MatAB 5/1983, S. 6
}

cher Gliederung sind es die bei Beschäftigung und Arbeitslosigkeit überaus starken saisonalen Schwankungen ausgesetzten Berufe

Pflanzenbauer, einschl. Gartenbauer, Forst- und Jagdberufe, Tierzüchter und Fischereiberufe,

die mit 2,13 die höchste Mehrfacharbeitslosigkeit überhaupt aufweisen. In regionaler Gliederung findet dies seinen Niederschlag in überdurchschnittlich hoher Mehrfacharbeitslosigkeit in Schleswig-Holstein/Hamburg, in BadenWürttemberg und Bayern (bei ansonsten vergleichsweise günstiger Arbeitsmarktlage in den südlichen Bundesländern). Dies überrascht nicht. Aus einer früheren Untersuchung ist nämlich bekannt, daß in Bayern fast $20 \%$ der durchschnittlichen Arbeitslosigkeit der Saisonarbeitslosigkeit zuzurechnen ist, die Anfang der 80er Jahre im Bundesgebiet lediglich $9,1 \%$ betrug. ${ }^{31}$ ) Aus dieser Untersuchung ist auch bekannt, daß über $80 \%$ der Saisonarbeitslosen (mit regelmäßiger Unterbrechung der Erwerbstätigkeit) Männer mittleren Alters sind. Damit dürfte auch ein Teil des Unterschiedes in der Mehrfacharbeitslosigkeit zwischen Männern und Frauen zu erklären sein. Allerdings kann nicht übersehen werden, daß saisonale Arbeitslosigkeit seit langem rückläufig ist. Ihr Anteil an der Gesamtarbeitslosigkeit beträgt gegenwärtig nur noch $6 \% .{ }^{32}$ ) Insoweit dürfte auch ihr Beitrag zu Mehrfacharbeitslosigkeit insgesamt nicht dominierend sein. Auch wird Arbeitslosigkeit saisonaler Art, die in bestimmten Berufen und Regionen schon fast Bestandteil der Erwerbsbiographie der Betroffenen ist, nicht so schwerwiegend empfunden und beurteilt wie andere Formen der Arbeitslosigkeit.

\subsubsection{Mehrfacharbeitslosigkeit bei bestimmten demographischen Gruppen}

Es wurde oben schon ausgeführt, daß man über eine lange Zeit hinweg wie selbstverständlich davon ausging, daß besondere Personengruppen, die sich in Zugangs- und Verweilrisiken in die bzw. in der Arbeitslosigkeit deutlich voneinander unterscheiden, auch im Hinblick auf Mehrfacharbeitslosigkeit und kumulative Arbeitslosigkeit zu bestimmen wären.

Höhere Mehrfacharbeitslosigkeit in Verbindung mit kürzeren Dauern erwartete man insbesondere bei Jüngeren (gegenüber Älteren) oder bei gering Qualifizierten gegenüber höher Qualifizierten. Die ersten Erhebungen bestätigten diese Vermutungen und verfestigten $\operatorname{sie}^{33}$ ). Dagegen wird in nahezu allen hier zitierten Studien - offenbar als Effekt der Überraschung - hervorgehoben, daß Mehrfacharbeitslosigkeit sich eher gleichmäßig über demographische Gruppen hinweg verteile, und daß insbesondere alle Altersgruppen von Mehrfacharbeitslosigkeit betroffen seien. Die eigenen Daten bestätigen dies weitgehend.

Der Versuch einer Erklärung dieser Differenzen ist weitgehend spekulativ. Zunächst ist einmal festzuhalten, daß die Unterschiede bei bestimmten Personengruppen nicht völlig eingeebnet sind, sondern eben nur weit geringer als früher bzw. als erwartet. So haben Männer - auch wegen der Saisonarbeitslosigkeit - eine nach wie vor höhere Mehrfacharbeitslosigkeit als Frauen; eine höhere Qualifikation, wie in den qualifizierten Fertigungs- und Dienstleistungsberufen und im Berufsbereich der Technischen Berufe (Tabelle 1), tritt mit zum Teil deutlich geringerer Mehrfacharbeitslosigkeit in Erscheinung. Eine Verbleibsanalyse von Arbeitslosen (Zugänge im November 1981) zeigt die gleiche Tendenz $\left.{ }^{34}\right)$. 
Zum zweiten dürften die früher fast durchweg kurzen Beobachtungszeiträume, die inzwischen durch deutlich längere abgelöst wurden, eine Verzerrung hervorgerufen haben; dies insoweit, als in einem kurzen Beobachtungszeitraum Mehrfacharbeitslosigkeit nur bei kurzen Dauern auftreten kann und beobachtbar ist. Längere Arbeitslosigkeitsdauern, wie sie insbesondere bei älteren Arbeitslosen auftreten, können als Mehrfacharbeitslosigkeit dabei überwiegend nicht erfaßt werden.

Drittens ist es durchaus möglich, daß die inzwischen in praktisch allen Industriestaaten langdauernde Massenarbeitslosigkeit zu einer „Strukturalisierung“ geführt hat, die sich in einer Konzentration der Arbeitslosigkeit auf eine Gruppe mit langer Dauer und eine andere mit instabilen Beschäftigungsverhältnissen niederschlägt. Auf dieses Problem wird im folgenden noch näher eingegangen.

\subsubsection{Destabilisierung nach Arbeitslosigkeit}

Von einigen Autoren, deren Arbeiten hier einbezogen wurden, wurde mit Nachdruck hervorgehoben, daß der Grund für Mehrfacharbeitslosigkeit in erster Linie eine bereits eingetretene Arbeitslosigkeit sei. Auch andere Indikatoren, z.B. die Dauer des vorausgegangenen Beschäftigungsverhältnisses und die unterschiedlichen Risiken bei Zugängern/Abgängern/Beständen an Arbeitslosen lassen sich auf diesen Nenner bringen. Begründet wird dies vor allem mit dem Prinzip „last in, first out": „Zusammengenommen deuten die Befunde darauf hin, daß das hohe Mehrfacharbeitslosigkeitsrisiko und der infolgedessen erhebliche Umfang instabiler Wiedereingliederungsverläufe bei den ehemaligen Arbeitslosen in erster Linie auf den Verlust mit der Betriebszugehörigkeitsdauer verknüpfter Senioritätsrechte, speziell des erhöhten Kündigungsschutzes, zurückzuführen sind.“(35)

Daneben werden bei längerer (auch kumulativer) Arbeitslosigkeit der Verlust von Kenntnissen und Fertigkeiten und allgemein auch die durch Arbeitslosigkeit eingetretene Stigmatisierung angeführt.

Die hier vorgenommene Untersuchung, in der ein gegebenes Arbeitslosigkeitsvolumen auf Personen und Fälle verteilt und in welcher die Mehrfacharbeitslosigkeit und kumulative Arbeitslosigkeit indirekt geschätzt wurden, läßt eine direkte Überprüfung dieser Hypothesen zunächst nicht zu. Das dem IAB zugängliche Datenmaterial hat aber den Vorteil, unterschiedlich lange Zeiträume und auch die Entwicklung der Mehrfacharbeitslosigkeit im Zeitverlauf analysieren zu können. Setzt man die Hypothese, daß eingetretene Arbeitslosigkeit die Wahrscheinlichkeit nachfolgender Arbeitslosigkeit erhöht, als richtig voraus, so muß sich im Zeitverlauf - bei gleichbleibend schlechter Arbeitsmarktlage - die Mehrfacharbeitslosigkeit ständig erhöhen.

Betrachtet man zu diesem Zweck die Verteilung der Arbeitslosigkeit (bzw. der Zeiten des Leistungsbezugs) auf Personen und Fälle in den 5-Jahreszeiträumen 1977-1981 und 1982-1986 (Tabelle 9), so sieht man, daß die Mehrfacharbeitslosigkeit, also z.B. die Zahl der arbeitslosen Personen, die im Beobachtungszeitraum mehr als eine Arbeitslosigkeitsperiode erleben (hier: Leistungsperiode), zugenommen hat.

${ }^{35}$ ) Büchtemann, C.F., Rosenbladt, B. von, Kumulative Arbeitslosigkeit, a.a.O., S. 270
Tabelle 9: Häufigkeit und Dauer von Mehrfacharbeitslosigkeit bzw. von Leistungsperioden in 5-Jahreszeiträumen

\begin{tabular}{|c|c|c|c|}
\hline $\begin{array}{c}\text { Zahl der } \\
\text { Personen } \\
(1000)\end{array}$ & $\begin{array}{c}\text { Arbeitslosig- } \\
\text { keitsperioden } \\
\text { bzw. Leistungs- } \\
\text { perioden }\end{array}$ & $\begin{array}{l}\text { durchschnitt- } \\
\text { liche Dauer } \\
\text { pro Periode } \\
\text { (Wochen) }\end{array}$ & $\begin{array}{l}\text { durchschnitt- } \\
\text { liche Dauer } \\
\text { pro Person } \\
\text { (Wochen) }\end{array}$ \\
\hline \multicolumn{4}{|c|}{ 1/1977 bis $12 / 1981$} \\
\hline 3940,1 & 1 & 21,1 & 21,1 \\
\hline 1411,0 & 2 & 15,6 & 31,2 \\
\hline 563,8 & 3 & 13,3 & 40,0 \\
\hline 252,8 & 4 & 11,9 & 47,8 \\
\hline 139,6 & 5 & 11,0 & 54,9 \\
\hline 51,3 & 6 & 9,6 & 57,6 \\
\hline 20,1 & 7 & 8,5 & 59,2 \\
\hline 8,4 & 8 & 7,5 & 60,4 \\
\hline 3,5 & 9 & 7,0 & 63,3 \\
\hline 1,7 & 10 & 6,5 & 64,6 \\
\hline 2,4 & mehr als 10 & 3,5 & 67,0 \\
\hline 6394,6 & & 16,2 & 27,3 \\
\hline \multicolumn{4}{|c|}{$1 / 1982$ bis $12 / 1986$} \\
\hline 4523,0 & 1 & 35,3 & 35,3 \\
\hline 1834,0 & 2 & 23,3 & 46,7 \\
\hline 791,5 & 3 & 18,9 & 56,7 \\
\hline 365,0 & 4 & 15,9 & 63,6 \\
\hline 202,2 & 5 & 13,7 & 68,4 \\
\hline 71,1 & 6 & 11,8 & 70,9 \\
\hline 26,4 & 7 & 10,3 & 72,0 \\
\hline 10,5 & 8 & 8,9 & 71,3 \\
\hline 4,7 & 9 & 7,8 & 70,0 \\
\hline 2,4 & 10 & 6,8 & 67,6 \\
\hline 3,3 & mehr als 10 & 3,6 & 69,9 \\
\hline 7834,1 & & 24,2 & 42,8 \\
\hline
\end{tabular}

Im Zeitraum 1977 bis 1981 hatten von den über 6 Mio. Leistungsempfängern $61,6 \%$ gerade eine Leistungsperiode; im Zeitraum 1982 bis 1986 waren es dagegen nur noch $57,7 \%$. Betrachtet man die prozentualen Veränderungen der Personenzahlen (Tabelle 10), so fällt auf, daß sich bei einem Anstieg der Zahl der Personen um insgesamt $22,5 \%$ diejenigen mit nur einer Periode lediglich um $14,8 \%$ erhöhte. Die Zahl der Personen mit zwei Perioden erhöhte sich um 30,0\%, mit drei Perioden um 40,4\%, mit vier Perioden um $44,3 \%$ usw. Es findet also eine Verschiebung innerhalb der Verteilung statt hin zu den Personen mit mehreren Arbeitslosigkeits- bzw. Leistungsperioden.

Tabelle 10: Veränderungsraten bei Leistungsempfängern und Leistungsperioden 1982/86 gegenüber 1977/81

\begin{tabular}{|c|c|c|}
\hline \multirow[t]{2}{*}{$\begin{array}{c}\text { Leistungsempfänger } \\
\text { mit ..... } \\
\text { Leistungsperioden }\end{array}$} & $\begin{array}{l}\text { Leistungs- } \\
\text { empfänger } \\
\text { (Personen) }\end{array}$ & $\begin{array}{c}\text { Dauer } \\
\text { pro } \\
\text { Leistungsfall }\end{array}$ \\
\hline & \multicolumn{2}{|c|}{$\%$} \\
\hline 1 & $+14,8$ & $+67,3$ \\
\hline 2 & $+30,0$ & $+49,4$ \\
\hline 3 & $+40,4$ & $+42,1$ \\
\hline 4 & $+44,3$ & $+33,6$ \\
\hline 5 & $+50,7$ & $+24,5$ \\
\hline 6 & $+38,8$ & $+22,9$ \\
\hline 7 & $+31,5$ & $+21,2$ \\
\hline 8 & $+25,1$ & $+18,7$ \\
\hline 9 & $+32,0$ & $+11,4$ \\
\hline 10 & $+42,3$ & $+4,6$ \\
\hline Gesamt & $+22,5$ & $+49,4$ \\
\hline
\end{tabular}


Nun könnte dies auch ausschließlich auf der veränderten Arbeitsmarktsituation im zweiten Zeitraum gegenüber dem ersten beruhen, oder mit anderen Worten, die Personen in beiden Zeiträumen könnten völlig verschieden sein, womit dann die aufgestellte Hypothese nicht einmal indirekt zu belegen wäre. Die Auswertung des Zehn-Jahreszeitraumes von 1977 bis 1986 (Tabelle 5) zeigt aber eine noch deutlichere Konzentration der Personen auf die Kategorien mit zwei und mehr Perioden. Nur noch 54,2\% der Leistungsempfänger haben in diesem Zeitraum eine Leistungsperiode. Dies ist aber nur möglich, wenn die Personen in beiden Zeiträumen wenigstens teilweise identisch sind.

Damit zeigt sich aber die Grundtendenz der zunehmenden Konzentration von Arbeitslosigkeit bei bestimmten Personen. Anderwärts gemachte Äußerungen derart, eine einmal eingetretene Arbeitslosigkeit sei die Hauptursache für erneute Arbeitslosigkeit, werden indirekt bestätigt. Dies hat aber in gewisser Weise fatale Konsequenzen für arbeitsmarktpolitische Prophylaxe und Therapie, insbesondere auch für die Arbeitsvermittlung und Arbeitsberatung. Erstens sind Mehrfacharbeitslosigkeit und kumulative Arbeitslosigkeit keine vernachlässigenswerten, weil nicht nur übergangsweise auftretenden Erscheinungen. Zweitens werden sie erst erkennbar, wenn eine Erwerbsbiographie bereits instabil geworden ist. Das erschwert prophylaktische Maßnahmen erheblich, weil nur wenige persönliche Merkmale den Kreis der potentiell von Mehrfacharbeitslosigkeit betroffenen Personen unscharf beschreiben können.

\section{Schlußbetrachtungen}

Die hier vorgestellte Untersuchung zeigt - trotz aller Einschränkungen aus den Restriktionen der Datenbasis - weitgehende Übereinstimmung mit Ergebnissen anderer Studien.

Sieht man einmal von den methodischen Problemen im Hinblick auf Definition und Messung von Mehrfacharbeitslosigkeit und kumulativer Arbeitslosigkeit ab, so ist wohl eine wichtige Feststellung die, daß die Bedeutung persönlicher demographischer Merkmale/Eigenschaften im Hinblick auf die Wahrscheinlichkeit, Mehrfacharbeitslosigkeit zu erfahren, vergleichsweise gering ist oder geringer geworden ist.

Durch die in dieser Untersuchung verwendeten längeren Beobachtungszeiträume (sie helfen statistische Artefakten, die sich bei Verwendung kürzerer Zeiträume ergeben, zu beseitigen) läßt sich zeigen, daß die lange anhaltende hohe Arbeitslosigkeit auch zu einer Strukturalisierung bei den Arbeitslosen führt, die man auf den ersten Blick und unter Verwendung üblicher Statistiken als eher unproblematisch einstufen würde. Aus eben diesen Statistiken wissen wir, daß etwa zwei Drittel der Arbeitslosen nach einer vergleichsweise kurzen Dauer von unter sechs Monaten schon wieder in Arbeit einmünden. Aber gerade in der Gruppe dieser Arbeitslosen, die relativ schnell wieder eine Arbeitsstelle finden, steckt offenbar der Kern jener, die nach kurzer Zeit erneut arbeitslos werden. Eine zweite wichtige Erkenntnis ist die, daß die isoliert ermittelte oder ausgewiesene Mehrfacharbeitslosigkeit ohne Kenntnis der kumulativen Arbeitslosigkeit manchmal zu Fehlinterpretationen führt. So wird die deutlich höhere Mehrfacharbeitslosigkeit der Männer gegenüber der der Frauen durch kumulative Arbeitslosigkeit völlig ausgeglichen; in regionaler Hinsicht würde die hohe Mehrfacharbeitslosigkeit in Baden-Württemberg gegenüber der eher geringeren in Nordrhein-Westfalen die tatsächliche Situation am Arbeitsmarkt geradezu auf den Kopf stellen. Die geringere Belastung Qualifizierter durch Arbeitslosigkeit kommt in der kumulativen Arbeitslosigkeit erst richtig zum Vorschein. Die Mehrfacharbeitslosigkeit allein könnte hier z.T. sogar zu Fehlschlüssen führen. Auch bei etwa gleicher Mehrfacharbeitslosigkeit im Hinblick auf Altersgruppen eine der Überraschungen dieser und der hier zitierten Untersuchungen - zeigt Mehrfacharbeitslosigkeit allein bei weitem nicht die besonderen Problemlagen der Älteren auf. Bei gleich hoher Mehrfacharbeitslosigkeit haben sie, wegen der deutlich längeren Dauer einzelner Perioden, eine fast doppelt so hohe kumulative Arbeitslosigkeit wie die Jüngeren. Sie sind mithin bei eingetretener Arbeitslosigkeit in allen Aspekten deutlich benachteiligt. Mehrfacharbeitslosigkeit und kumulative Arbeitslosigkeit sollten deshalb immer zusammen ermittelt und ausgewiesen werden.

Der Umfang der Mehrfacharbeitslosigkeit wurde als statistischer Mittelwert mit 1,7 angegeben; für den gewählten Fünf-Jahreszeitraum haben also durchschnittlich 100 arbeitslose Personen 170 Arbeitslosigkeitsperioden. (Ein ähnliches Ergebnis zeigt auch die Österreich-Studie.) Die Verteilung dieser Perioden ist mit dem gegenwärtigen Material nicht zu ermitteln; sie liegt nur für Leistungsempfänger vor und hier mit der Tendenz, Mehrfacharbeitslosigkeit zu gering auszuweisen. (Wird ein Arbeitsloser, der nach Leistungserschöpfung wieder erwerbstätig wird, erneut arbeitslos, ohne neue Ansprüche erworben zu haben, erscheint diese Mehrfacharbeitslosigkeit in der Statistik der Leistungsempfänger nicht.) Wenn trotzdem nur wenig mehr als die Hälfte der im Fünf-Jahreszeitraum von Arbeitslosigkeit Betroffenen mit einer Leistungsperiode in Erscheinung treten, die übrigen aber mit zwei und mehr Perioden, so bestätigen auch diese Zahlen die DIWErgebnisse, wonach jeder zweite (der im Panel beobachteten Probanden) nach drei Jahren noch oder wieder arbeitslos ist.

Dieser Umfang der Mehrfacharbeitslosigkeit bzw. kumulativer Arbeitslosigkeit oder, was dafür steht, destabilisierter Erwerbsverläufe, sollte es daher nahelegen, Aussagen zur Arbeitslosigkeit noch differenzierter zu formulieren. Daß $70 \%$ der Arbeitslosen die Arbeitslosigkeit mit der Aufnahme einer Arbeit beenden, müßte ergänzt werden um einen Hinweis darauf, daß für viele von ihnen damit allerdings keine stabile Wiedereingliederung ins Erwerbsleben gelungen ist.

$\mathrm{Da}$ die Arbeitslosen keinen „festen Block“ darstellen (eine Kernaussage des IAB), müßte ergänzt werden um den Hinweis, daß aber ein erheblicher Teil von ihnen - im Fünf-Jahreszeitraum rund $50 \%$ - nach Arbeitslosigkeit erneut arbeitslos werden. Aus einer anderen Sichtweise findet also doch Strukturalisierung statt, die zu einer Art unscharfem „festen Block“ instabil Beschäftigter führt. Letztlich zeigt sich hier auch die Kehrseite einer Medaille, die aus der Linderung akuter Arbeitsmarktprobleme durch Maßnahmen oder befristete Beschäftigungsverhältnisse usw. resultiert. Die in der kurzen Frist oder Sicht sowohl individuell wie gesellschaftlich enorm hilfreichen und hilfreich empfundenen Möglichkeiten, wenigstens befristet (,besser befristet beschäftigt als unbefristet arbeitslos") in eine Maßnahme eintreten oder eine Beschäftigung aufnehmen zu können, sind eben auch ein Teil der nicht dauerhaft gelungenen Reintegration.

Solche Aspekte der Mehrfacharbeitslosigkeit werden in Zukunft sicher ausführlicher und vertiefter diskutiert werden müssen. 